\title{
Space and time error estimates for a first order, pressure stabilized finite element method for the incompressible Navier-Stokes equations
}

\author{
J. Blasco ${ }^{\mathrm{a}, *}$, R. Codina ${ }^{\mathrm{b}}$ \\ a Departament de Matemàtica Aplicada I, ETSEIB, Universitat Politècnica de Catalunya, Campus Sud, Edifici H, \\ Avgda. Diagonal 647, 08028 Barcelona, Spain \\ ${ }^{\mathrm{b}}$ Departament de Resistència de Materials i Estructures a l'Enginyeria, ETSECCPB, Universitat Politècnica de Catalunya, \\ Campus Nord, Edifici C1, c/ Jordi Girona 1-3, 08034 Barcelona, Spain
}

Received 5 November 1999; received in revised form 6 April 2001; accepted 6 April 2001

\begin{abstract}
In this paper we analyze a pressure stabilized, finite element method for the unsteady, incompressible NavierStokes equations in primitive variables; for the time discretization we focus on a fully implicit, monolithic scheme. We provide some error estimates for the fully discrete solution which show that the velocity is first order accurate in the time step and attains optimal order accuracy in the mesh size for the given spatial interpolation, both in the spaces $L^{2}(\Omega)$ and $H_{0}^{1}(\Omega)$; the pressure solution is shown to be order $\frac{1}{2}$ accurate in the time step and also optimal in the mesh size. These estimates are proved assuming only a weak compatibility condition on the approximating spaces of velocity and pressure, which is satisfied by equal order interpolations. $\odot 2001$ IMACS. Published by Elsevier Science B.V. All rights reserved.
\end{abstract}

Keywords: Finite elements; Incompressible flow; Pressure instability; Navier-Stokes equations; Error analysis

\section{Introduction}

The purpose of this paper is to provide some error estimates for a pressure stabilized, finite element method for the numerical solution of the unsteady, incompressible Navier-Stokes equations in the primitive variables velocity and pressure. The method was introduced in [7] as an extension to the transient case of a technique initially developed for the Stokes problem [5] and then extended to the steady, incompressible Navier-Stokes equations [6].

\footnotetext{
* Corresponding author.

E-mail addresses: blasco@ma1.upc.es (J. Blasco), ramon.codina@upc.es (R. Codina).

URLs: http://www-ma1.upc.es/ blasco (J. Blasco), http://www.rmee.upc.es/homes/codina (R. Codina).
} 
The stabilization of the pressure in incompressible flow problems has received much attention in the last decades. Numerical schemes have been developed which bypass the need for the approximating spaces of velocity and pressure to satisfy the compatibility condition met when using standard Galerkin methods. Stabilized formulations were first introduced under the idea of Petrov-Galerkin methods [17], which then led to Galerkin Least Squares (GLS) techniques. These were first developed in the context of advection-diffusion equations [18], and then extended to the linearized, steady incompressible NavierStokes equation in [9] (see also [10] and the references therein). More recently, the GLS technique has evolved into the idea of subgrid-scale models (see [4,16]). All these techniques have been analyzed in the literature for steady problems using arbitrary finite element interpolations. Error analysis both in space and time for stabilized formulations of transient problems have been given in [19], for advectiondiffusion problems, and [13], for the incompressible Navier-Stokes equations. In this last reference, the analysis was based on the assumption that the time step $\delta t$ is of the same order as the mesh size $h: \delta t \simeq h$. Moreover, it was restricted to the case of piecewise linear elements.

On the other hand, some combinations of finite element spaces which satisfy the discrete compatibility condition have been analyzed for the Stokes problem and proven to be stable and yield optimal order accuracy of the solution (see, for instance, [3,22]). Assuming a (mixed) finite element pair which satisfies the discrete compatibility condition, some analysis of methods for the unsteady problem have been given: Heywood and Rannacher [14,15] proved second order error estimates
accuracy in the mesh size for a mixed method using a Crank-Nicholson
et al. [1] also proved second order estimates in time and optimal order
method under a stability restriction on the time step of the form $\delta t \leqslant$
space; finally, Guermond and Quartapelle [12] analyzed the classical
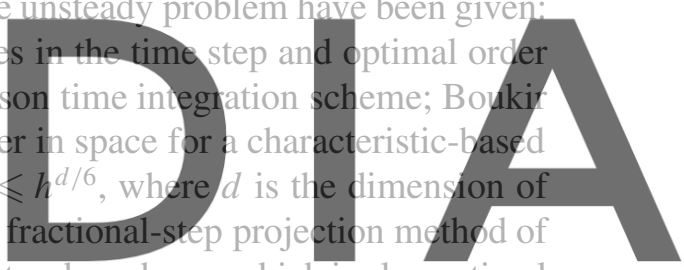

A.J. Chorin and R. Temam in its incremental form, which yields a first order scheme which is also optimal in space (a second order method can also be developed). Their analysis is based on the satisfaction of the

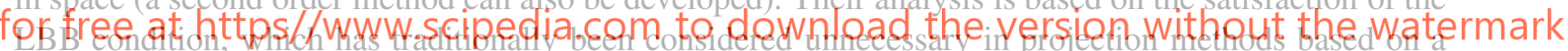

Poisson equation for the pressure. This condition can be avoided assuming $\delta t \geqslant h^{l+1}$, where $l$ is the order of the spatial interpolation, in the stability analysis of the non-incremental form of the method, but not in the convergence one.

We analyze here a stabilized formulation of the unsteady problem which employs a finite element, pressure gradient projection technique [6] and a fully implicit, backward Euler scheme for the time integration. We show that first order accuracy in time is maintained in the fully discrete method, which attains optimal order accuracy in space for the given interpolation. The analysis is carried out assuming only a weak compatibility condition on the approximating spaces of velocity and pressure, which was proven to be satisfied by simplicial equal order finite element interpolations in [5]. The error estimates obtained are given in terms of a certain norm of the velocity in $L^{2}(\Omega)$ and $H_{0}^{1}(\Omega)$ and the pressure and its gradient in $L^{2}(\Omega)$. We first analyze the temporal error by considering a semidiscrete approximation of the problem, and then study the fully discrete method, with both a linearized and a nonlinear approximation of the convective term.

It has to be remarked that the purpose of the technique employed here is to stabilize the pressure solution; the instabilities due to the convective term at high cell Reynolds numbers are not addressed at with this formulation. Moreover, the interest here relies on showing how the technique that we use to stabilize the pressure, with respect to the spatial interpolation, can be analyzed in transient problems, regardless of the particular time integration method employed. We concentrate on a fully implicit, backward Euler scheme, which, although being only first order accurate, is unconditionally stable; 
however, other methods could also be considered (see [7]). The resulting scheme is computationally feasible (see [7]), and also suitable as an iterative method to reach steady states.

Our presentation is split into two sections. In Section 2 we state the problem to solve, recall some known properties of its solution and introduce some notation; we then present the semidiscrete approximation considered and finally the fully discrete, stabilized finite element method. In Section 3 we state and prove our error estimates, first for the semidiscrete and then for the fully discrete problems. We first recall a stability estimate which was proven in [7] under weak assumptions on the continuous solution; then we prove some optimal order error estimates for the velocity, from which we obtain an improved stability estimate as a side product. We finally analyze the pressure solution, for which we also obtain optimal order error estimates.

\section{Deseription of the method}

\subsection{Problem statement}

\section{The evolution of viscous, incompressible fluid flow in a bounded domain $\Omega \subset \mathbb{R}^{d}(d=2,3)$ is governed, in the primitive variable formulation, by the unsteady, incompressible
equations:
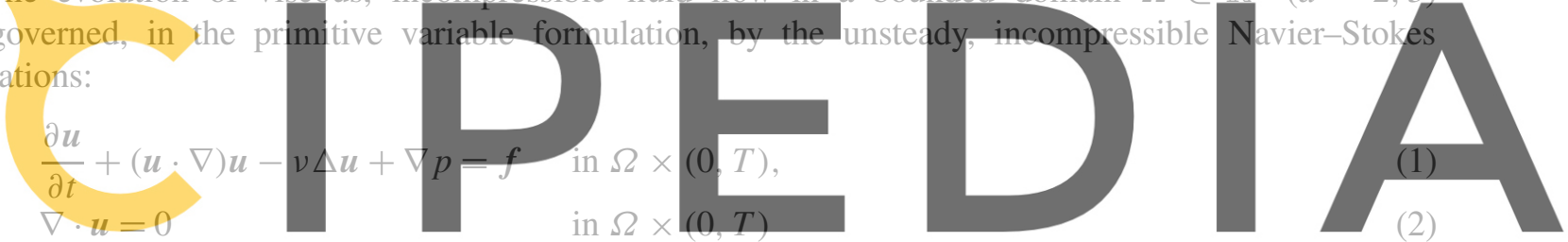

on $\Omega \times(0, T)$ (with $T>0$ a given final time), where $u(x, t) \in \mathbb{R}^{d}$ is the fluid velocity at position $x \in \Omega$

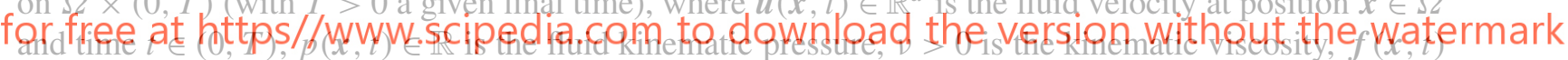
is an external force, $\nabla$ is the gradient operator, $\nabla$. is the divergence operator and $\Delta$ is the Laplacian operator (here, and in what follows, boldface characters denote vector quantities). Boundary conditions have to be given to complete the equation system (1)-(2). For the sake of simplicity, only homogeneous Dirichlet type boundary conditions are considered here:

$$
\boldsymbol{u}=\mathbf{0} \quad \text { on } \Gamma \times(0, T),
$$

where $\Gamma=\partial \Omega$. An initial condition must also be specified for the velocity:

$$
\boldsymbol{u}(\boldsymbol{x}, 0)=\boldsymbol{u}_{0}(\boldsymbol{x}) \text { in } \Omega .
$$

The treatment of the above equations of motion requires of the usual Sobolev spaces $H^{m}(\Omega), m \geqslant 0$, consisting of functions with distributional derivatives up to order $m$ belonging to $L^{2}(\Omega)$. The scalar product in $H^{m}(\Omega)$ is denoted by $(u, v)_{m}$ (the subscript $m$ may be omitted when it equals 0 ) and its norm by $\|u\|_{m}$. The closed subspaces $H_{0}^{1}(\Omega)$, consisting of functions in $H^{1}(\Omega)$ with zero trace on $\Gamma$, and $L_{0}^{2}(\Omega)$, made up with functions in $L^{2}(\Omega)$ with zero mean on $\Omega$, will also be needed. Also, let $H^{-1}(\Omega)$ denote the dual space of $H_{0}^{1}(\Omega)$, the duality between these two spaces being denoted by $\langle$,$\rangle , and let:$

$$
W=\left\{\boldsymbol{u} \in \boldsymbol{H}_{0}^{1}(\Omega) \mid \nabla \cdot \boldsymbol{u}=0\right\} .
$$

Assuming $\boldsymbol{u}_{0} \in \boldsymbol{H}_{0}^{1}(\Omega)$ and $\boldsymbol{f} \in L^{2}\left(0, T ; \boldsymbol{H}^{-1}(\Omega)\right)$, and if $\Omega$ is bounded and Lipschitz continuous, problem (1)-(4) has at least one solution $\boldsymbol{u} \in L^{\infty}\left(0, T ; \boldsymbol{L}^{2}(\Omega)\right) \cap L^{2}\left(0, T ; \boldsymbol{H}_{0}^{1}(\Omega)\right.$ ) (see [24]). 
Uniqueness and more regularity of the solution can be achieved by assuming more regularity on $f$, $\boldsymbol{u}_{0}$ and $\Omega$. In particular, we assume hereafter that the continuous solution $(\boldsymbol{u}, p)$ of (1)-(4) is unique and satisfies:

(R1) $\boldsymbol{u} \in L^{\infty}\left(0, T ; \boldsymbol{H}^{2}(\Omega)\right) \cap C^{0}(0, T ; W), p \in L^{\infty}\left(0, T ; H^{1}(\Omega)\right) \cap C^{0}\left(0, T ; L_{0}^{2}(\Omega)\right)$,

(R2) $\boldsymbol{u}_{t} \in L^{2}\left(0, T ; \boldsymbol{L}^{2}(\Omega)\right)$,

(R3) $\int_{0}^{T} t\left\|\boldsymbol{u}_{t t}(t)\right\|_{-1}^{2} \mathrm{~d} t \leqslant C$,

(R4) $\int_{0}^{T}\left\|\boldsymbol{u}_{t t}(t)\right\|_{W^{\prime}}^{2} \mathrm{~d} t \leqslant C$.

The subscript $t$ is employed hereafter for $\partial / \partial t$, and we use $C$ as a generic constant depending of $\boldsymbol{f}, \boldsymbol{u}_{0}$, $\Omega$ and $v$, but not on the time step $\delta t$ nor on the mesh size $h$; also, $W^{\prime}$ is the dual space of $W$. Sufficient conditions for (R1)-(R3) to hold can be found [14]; for (R4), see [20,21]. In particular, it is required that $f \in L^{2}\left(0, T ; \boldsymbol{L}^{2}(\Omega)\right)$, which we assume from now on.

Let us call $V=\boldsymbol{H}_{0}^{1}(\Omega)$ and $Q=L_{0}^{2}(\Omega)$. In what follows the following notation will be used for the weak form of the different terms in Eqs. (1)-(2):

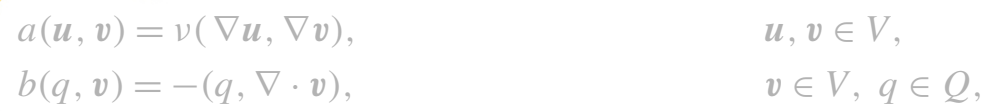

All these forms are contin arising from the convective incompressibility conditio term in (1). Besides, a exists a constant $K_{a}>$
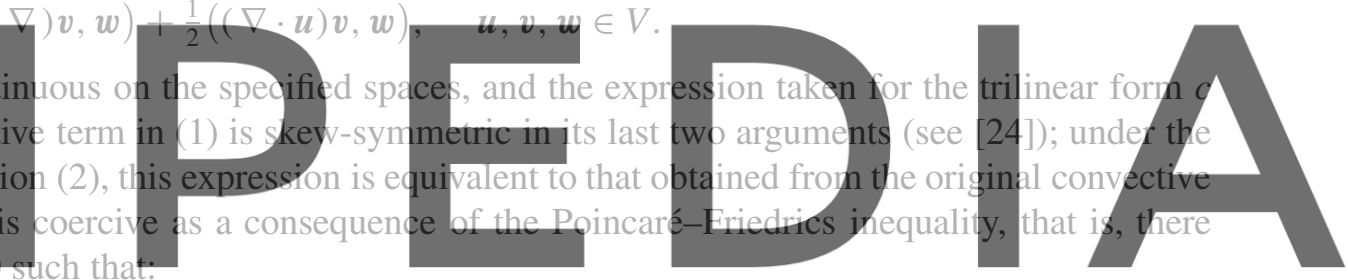

\section{free atu hit}

and $b$ satisfies the (continuous) inf-sup condition, that is, there exists a constant $K_{b}>0$ such that:

$$
\inf _{q \in Q}\left(\sup _{\boldsymbol{v} \in V} \frac{b(q, \boldsymbol{v})}{\|\boldsymbol{v}\|_{1}\|q\|_{0}}\right) \geqslant K_{b}>0
$$

(infima and suprema are always taken with respect to nonzero functions). Condition (5) is usually referred to as the inf-sup or LBB condition, after the work of O.A. Ladyzhenskaya, I. Babuška and F. Brezzi. Finally, $c$ satisfies other continuity properties, some of which are (see, e.g., [8]):

$$
c(\boldsymbol{u}, \boldsymbol{v}, \boldsymbol{w}) \leqslant\left\{\begin{array}{l}
C\|\boldsymbol{u}\|_{1}\|\boldsymbol{v}\|_{1}\|\boldsymbol{w}\|_{1} \\
C\|\boldsymbol{u}\|_{0}\|\boldsymbol{v}\|_{2}\|\boldsymbol{w}\|_{1} \\
C\|\boldsymbol{u}\|_{2}\|\boldsymbol{v}\|_{1}\|\boldsymbol{w}\|_{0} \\
C\|\boldsymbol{u}\|_{0}\|\boldsymbol{v}\|_{1}\|\boldsymbol{w}\|_{L^{\infty}(\Omega)} .
\end{array}\right.
$$

\subsection{Finite element approximation}

The numerical approximation of problem (1)-(2) that we analyze here was introduced in [7] as an extension to the transient case of a finite element method originally developed for steady problems. It is well known that discrete approximations of incompressible flow problems in primitive variables are restricted by the discrete inf-sup condition, that is, the discrete counterpart of condition (5); this prevents the use of many simple finite element combinations for the discrete spaces of the velocity and 
the pressure, such as equal order ones. The methods based on a pressure gradient projection circumvent this restriction by introducing the projection of the gradient of the discrete pressure onto the space of discrete velocities as a new variable of the problem; this allows, in particular, the use of equal order interpolations.

In the transient case, this methodology can be applied together with different time integration schemes; we concentrate here on an implicit, monolithic scheme using the trapezoidal rule, but extensions to other schemes such as fractional-step or multistep methods can be derived in a similar way (see [7] for a description of some of them).

\subsubsection{Semidiscrete problem}

We consider a parameter $\theta \in(0,1]$ and discretize Eqs. (1)-(2) in time first by the following implicit scheme, which we write in variational form: given a time step size $\delta t>0$, let $N=[T / \delta t]-1$; for $n \in\{0, \ldots, N\}$, let $t_{n}=n \delta t$; given $\boldsymbol{u}^{n} \in V$ and $p^{n} \in Q$, approximations of $\boldsymbol{u}\left(t_{n}\right)$ and $p\left(t_{n}\right)$, respectively, find $\boldsymbol{u}^{n+1} \in V$ and $p^{n+1} \in Q$ such that:

$$
\left(\frac{\boldsymbol{u}^{n+1}-\boldsymbol{u}^{n}}{\delta t}, \boldsymbol{v}\right)+c\left(\boldsymbol{u}^{n+\varepsilon \theta}, \boldsymbol{u}^{n+\theta}, \boldsymbol{v}\right)+a\left(\boldsymbol{u}^{n+\theta}, \boldsymbol{v}\right)+\left(\nabla p^{n+\theta}, \boldsymbol{v}\right),=\left(\boldsymbol{f}^{n+\theta}, \boldsymbol{v}\right),
$$
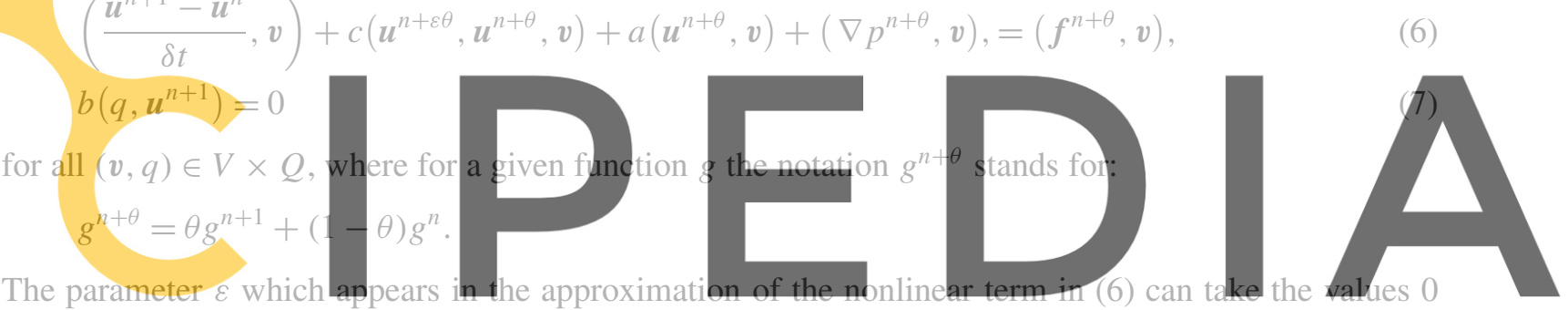

and 1 , corresponding to a linearized and a nonlinear approximation of convection, respectively. The first

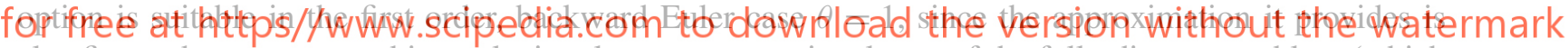
also first order accurate and it results in a lower computational cost of the fully discrete problem (which is then linear in each time step); the second option, however, enhances stability for highly convective flows and is compulsory in the Crank-Nicholson case $\theta=\frac{1}{2}$ to maintain second order accuracy. In this sense, we use the expression $c\left(\boldsymbol{u}^{n+\theta}, \boldsymbol{u}^{n+\theta}, \boldsymbol{v}\right)$ which differs from $(c(\boldsymbol{u}, \boldsymbol{u}, \boldsymbol{v}))^{n+\theta}$ (the form to which strict application of the trapezoidal rule would lead) by a second order term and is computationally simpler. Moreover, we assume that the semidiscrete pressures satisfy $\nabla p^{n+1} \in \boldsymbol{L}^{2}(\Omega)$; conditions on $\boldsymbol{f}$ and $\Omega$ for this assumption to hold can be found, for instance, in [11].

\subsubsection{Fully discrete method}

We now proceed to introduce a spatial approximation of the semidiscrete problem (6)-(7). Let $\Theta_{h}$ denote a finite element partition of the domain $\Omega$ of diameter $h$. We assume that all the element domains $K \in \Theta_{h}$ are the image of a reference element $\widehat{K}$ through polynomial mappings $\boldsymbol{F}_{K}$, affine for simplicial elements, bilinear for quadrilaterals and trilinear for hexahedra. On $\widehat{K}$ we define the polynomial spaces $R_{k}(\widehat{K})$ where, as usual, $R_{k}=P_{k}$ for simplicial elements and $R_{k}=Q_{k}$ for quadrilaterals and hexahedra. The finite element spaces we need are:

$$
\begin{aligned}
& Q_{h}=\left\{q_{h} \in C^{0}(\Omega) \cap L_{0}^{2}(\Omega) \mid q_{h \mid K}=\hat{q} \circ \boldsymbol{F}_{K}^{-1}, \hat{q} \in R_{k_{\mathrm{q}}}(\widehat{K}), K \in \Theta_{h}\right\}, \\
& V_{h}=\left\{\boldsymbol{v}_{h} \in\left(C^{0}(\Omega)\right)^{d} \mid \boldsymbol{v}_{h \mid K}=\hat{\boldsymbol{v}} \circ \boldsymbol{F}_{K}^{-1}, \hat{\boldsymbol{v}} \in\left(R_{k_{\mathrm{v}}}(\widehat{K})\right)^{d}, K \in \Theta_{h}\right\}, \\
& V_{h, 0}=\left\{\boldsymbol{v}_{h} \in V_{h} \mid \boldsymbol{v}_{h \mid \Gamma}=\mathbf{0}\right\} .
\end{aligned}
$$


Notice that both the velocity and pressure finite element spaces $V_{h, 0}$ and $Q_{h}$ are referred to the same partition and both are made up with continuous functions. These finite element spaces satisfy the following approximating properties (see, e.g., [23]): given $\boldsymbol{v} \in \boldsymbol{H}^{r}(\Omega), r \geqslant 2$, and $q \in H^{s}(\Omega), s \geqslant 1$, there exist $\Pi_{h, 1}(v) \in V_{h, 0}, \Pi_{h, 2}(q) \in Q_{h}$ and $\Pi_{h, 3}(\nabla q) \in V_{h}$ such that:

$$
\begin{aligned}
& \left\|\boldsymbol{v}-\Pi_{h, 1}(\boldsymbol{v})\right\|_{m_{1}} \leqslant C_{1} h^{k_{1}-m_{1}}\|\boldsymbol{v}\|_{k_{1}}, \\
& \left\|q-\Pi_{h, 2}(q)\right\|_{m_{2}} \leqslant C_{2} h^{k_{2}-m_{2}}\|q\|_{k_{2}}, \\
& \left\|\nabla q-\Pi_{h, 3}(\nabla q)\right\|_{m_{3}} \leqslant C_{3} h^{k_{3}-m_{3}}\|\nabla q\|_{k_{3}},
\end{aligned}
$$

for $0 \leqslant m_{i} \leqslant k_{i}(i=1,2,3)$, where:

$$
k_{1}=\min \left\{r, k_{\mathrm{v}}+1\right\}, \quad k_{2}=\min \left\{s, k_{\mathrm{q}}+1\right\}, \quad k_{3}=\min \left\{s-1, k_{\mathrm{v}}+1\right\} .
$$

Let now $\alpha>0$ be a given parameter. Given $\left(\boldsymbol{u}_{h}^{n}, p_{h}^{n}, \xi_{h}^{n}\right) \in V_{h, 0} \times Q_{h} \times V_{h}$, approximations of $\left(\boldsymbol{u}^{n}, p^{n}, \nabla p^{n}\right)$, we discretize (6)-(7) in space by finding $\left(\boldsymbol{u}_{h}^{n+1}, p_{h}^{n+1}, \xi_{h}^{n+1}\right) \in V_{h, 0} \times Q_{h} \times V_{h}$ such that:

$$
\begin{aligned}
& \left(\frac{\boldsymbol{u}_{h}^{n+1}-\boldsymbol{u}_{h}^{n}}{\delta t}, v_{h}\right)+c\left(\boldsymbol{u}_{h}^{n+\varepsilon \theta}, \boldsymbol{u}_{h}^{n+\theta}, v_{h}\right)+a\left(\boldsymbol{u}_{h}^{n+\theta}, v_{h}\right)+\left(\nabla p_{h}^{n+\theta}, v_{h}\right)=\left(f^{n+\theta}, v_{h}\right), \\
& -b\left(q_{h}, \boldsymbol{u}_{h}^{n+1}\right)+\alpha\left(\left(\nabla p_{h}^{n+1}, \nabla q_{h}\right)\right. \\
& -\left(\nabla p_{h}^{n+1}, \eta_{h}\right)+\left(\xi_{h}^{n+1}, \eta_{h}\right)=0 \\
& \text { all } \left.\left.\left(v_{h}, q_{h}, \eta_{h}\right) \in V_{h}, q_{h}\right)\right)=0 \\
& \text { projection of } \nabla p_{h}^{n+} \times \text { onto the space } V_{h} \text {; thus, the cases } \beta=0 \text { and } \beta=1 \text { correspond to an explicit } \\
& \text { an implicit approximation of the pressure gradient projection in the modified continuity equation (9), }
\end{aligned}
$$

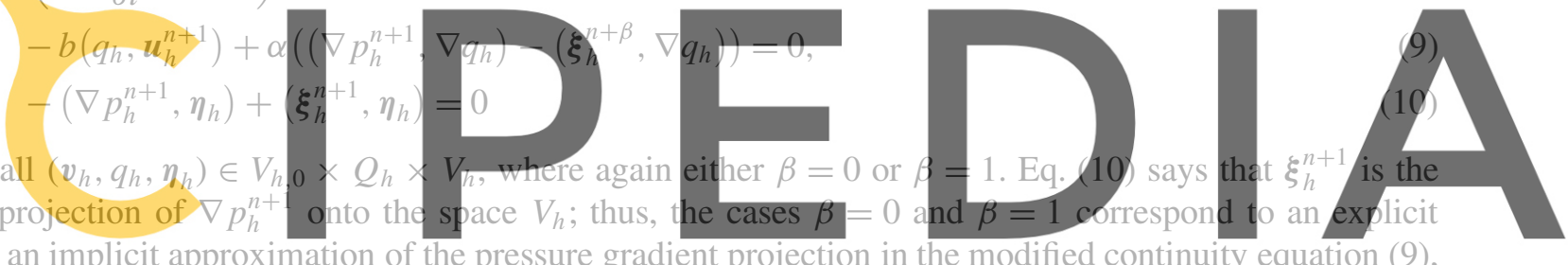

and an implicit approximation of the pressure gradient projection in the modified continuity equation (9),

domains; the numerical analysis of this method then requires of some regularity properties of the finite element mesh süch as its quasi-uniformity. However, this restriction can be relaxed by considering a set of elemental parameters $\alpha_{K}, K \in \Theta_{h}$, and replacing the $L^{2}$-scalar products appearing in (9)-(10) by a sum of products weighed in each element by $\alpha_{K}$. This extension to local parameters was analyzed in [6] for the steady, incompressible Navier-Stokes equations, and the analysis given there can be readily applied to the transient case. We restrict our attention here to the global parameter case to simplify the presentation.

\section{Stability and error analysis}

We now present a numerical analysis of the finite element method (8)-(10). For the time approximation, we restrict to the fully implicit, backward Euler case $\theta=1$, which is first order accurate in the time step. We split the errors of the method into a temporal error, due to the semidiscretization (6)-(7), and a spatial error, due to the stabilized, fully discrete method (8)-(10). In the case of study $\theta=1$, first order accuracy in the time step for the semidiscrete velocity solution can be shown by standard arguments; we include a proof of this result for completeness. We consider both the linearized method $\varepsilon=0$ and the fully nonlinear scheme $\varepsilon=1$. We then concentrate on the spatial approximation in the implicit pressure gradient case $\beta=1$. 


\subsection{Error estimates for the semidiscrete solution}

Let us define the continuous errors (as for the spatial variables) as

$$
\begin{aligned}
& \boldsymbol{e}_{c}^{n+1}=\boldsymbol{u}\left(t_{n+1}\right)-\boldsymbol{u}^{n+1}, \\
& r_{c}^{n+1}=p\left(t_{n+1}\right)-p^{n+1}, \\
& \boldsymbol{g}_{c}^{n+1}=\nabla r_{c}^{n+1} .
\end{aligned}
$$

We then have:

Theorem 1. Assume (R1), (R2) and (R4) hold. Then, there is a constant $C$ independent of $\delta t$ such that:

$$
\left\|\boldsymbol{e}_{c}^{N+1}\right\|_{0}^{2}+v \delta t \sum_{n=0}^{N}\left\|\boldsymbol{e}_{c}^{n+1}\right\|_{1}^{2} \leqslant C \delta t^{2} .
$$

\section{If $\varepsilon=1$, (11) holds for sufficiently small $\delta t$.}

Proof. We call $\boldsymbol{R}^{n}$ the truncation error defined by
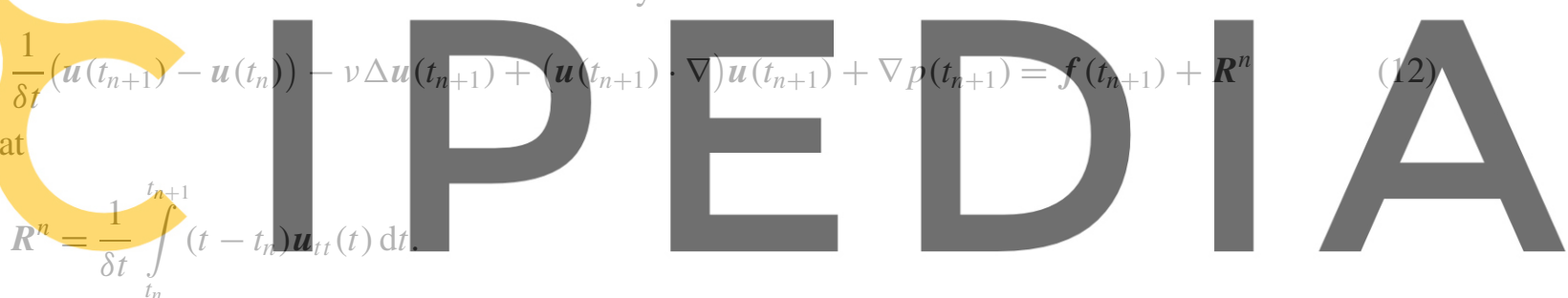

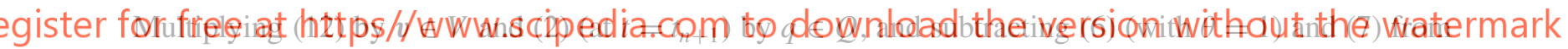

them, respectively, we find

$$
\begin{aligned}
& \left(\frac{\boldsymbol{e}_{c}^{n+1}-\boldsymbol{e}_{c}^{n}}{\delta t}, \boldsymbol{v}\right)+v\left(\nabla \boldsymbol{e}_{c}^{n+1}, \nabla \boldsymbol{v}\right)+\left(\nabla r_{c}^{n+1}, \boldsymbol{v}\right) \\
& \quad=\left\langle\boldsymbol{R}^{n}, \boldsymbol{v}\right\rangle+c\left(\boldsymbol{u}^{n+\varepsilon}, \boldsymbol{u}^{n+1}, \boldsymbol{v}\right)-c\left(\boldsymbol{u}\left(t_{n+1}\right), \boldsymbol{u}\left(t_{n+1}\right), \boldsymbol{v}\right), \\
& b\left(q, \boldsymbol{e}_{c}^{n+1}\right)=0 .
\end{aligned}
$$

Taking $\boldsymbol{v}=2 \delta t \boldsymbol{e}_{c}^{n+1}$ in (13) and $q=r_{c}^{n+1}$ in (14), and using the identity $(a-b, 2 a)=|a|^{2}-|b|^{2}+|a-b|^{2}$, we get

$$
\left\|\boldsymbol{e}_{c}^{n+1}\right\|_{0}^{2}-\left\|\boldsymbol{e}_{c}^{n}\right\|_{0}^{2}+\left\|\boldsymbol{e}_{c}^{n+1}-\boldsymbol{e}_{c}^{n}\right\|_{0}^{2}+2 \delta t v\left\|\nabla \boldsymbol{e}_{c}^{n+1}\right\|_{0}^{2}=2 \delta t\left\langle\boldsymbol{R}^{n}, \boldsymbol{e}_{c}^{n+1}\right\rangle+2 \delta t \mathrm{NLT}
$$

where NLT stands for

$$
\mathrm{NLT}=c\left(\boldsymbol{u}^{n+\varepsilon}, \boldsymbol{u}^{n+1}, \boldsymbol{e}_{c}^{n+1}\right)-c\left(\boldsymbol{u}\left(t_{n+1}\right), \boldsymbol{u}\left(t_{n+1}\right), \boldsymbol{e}_{c}^{n+1}\right) .
$$

For the Taylor residual term, one has (see, e.g., [20])

$$
2 \delta t\left\langle\boldsymbol{R}^{n}, \boldsymbol{e}_{c}^{n+1}\right\rangle \leqslant \frac{\delta t v}{3}\left\|\nabla \boldsymbol{e}_{c}^{n+1}\right\|_{0}^{2}+C \delta t^{2} \int_{t_{n}}^{t_{n+1}}\left\|\boldsymbol{u}_{t t}\right\|_{W^{\prime}}^{2} \mathrm{~d} t .
$$

The treatment of the NLT is different in the cases $\varepsilon=0$ and $\varepsilon=1$. 
Linearized case. When $\varepsilon=0$, we have

$$
\begin{aligned}
2 \delta t \mathrm{NLT} & =2 \delta t\left(c\left(\boldsymbol{u}^{n}, \boldsymbol{u}^{n+1}, \boldsymbol{e}_{c}^{n+1}\right)-c\left(\boldsymbol{u}\left(t_{n+1}\right), \boldsymbol{u}\left(t_{n+1}\right), \boldsymbol{e}_{c}^{n+1}\right)\right) \\
& =2 \delta t\left(-c\left(\boldsymbol{u}^{n}, \boldsymbol{e}_{c}^{n+1}, \boldsymbol{e}_{c}^{n+1}\right)-c\left(\boldsymbol{e}_{c}^{n}, \boldsymbol{u}\left(t_{n+1}\right), \boldsymbol{e}_{c}^{n+1}\right)-c\left(\boldsymbol{u}\left(t_{n+1}\right)-\boldsymbol{u}\left(t_{n}\right), \boldsymbol{u}\left(t_{n+1}\right), \boldsymbol{e}_{c}^{n+1}\right)\right) \\
& =T_{1}+T_{2}+T_{3},
\end{aligned}
$$

where $T_{1}=0$ due to the skew-symmetry of the trilinear form $c$, and, due to its continuity properties and the regularity property $(\mathrm{R} 1)$ of $\boldsymbol{u}$ :

$$
\begin{aligned}
T_{2} & =-2 \delta t c\left(\boldsymbol{e}_{c}^{n}, \boldsymbol{u}\left(t_{n+1}\right), \boldsymbol{e}_{c}^{n+1}\right) \\
& \leqslant C \delta t\left\|\boldsymbol{e}_{c}^{n}\right\|_{0}\left\|u\left(t_{n+1}\right)\right\|_{2}\left\|e_{c}^{n+1}\right\|_{1} \leqslant \frac{\delta t v}{3}\left\|\nabla \boldsymbol{e}_{c}^{n+1}\right\|_{0}^{2}+C \delta t\left\|\boldsymbol{e}_{c}^{n}\right\|_{0}^{2}, \\
T_{3} & =-2 \delta t c\left(\boldsymbol{u}\left(t_{n+1}\right)-u\left(t_{n}\right), u\left(t_{n+1}\right), \boldsymbol{e}_{c}^{n+1}\right) \\
& \leqslant C \delta t\left\|u\left(t_{n+1}\right)-u\left(t_{n}\right)\right\|_{0}\left\|u\left(t_{n+1}\right)\right\|_{2}\left\|e_{c}^{n+1}\right\|_{1} \leqslant \frac{\delta t v}{3}\left\|\nabla \boldsymbol{e}_{c}^{n+1}\right\|_{0}^{2}+C \delta t^{2} \int_{t_{n}}^{t_{n+1}}\left\|u_{t}\right\|_{0}^{2} \mathrm{~d} t .
\end{aligned}
$$

Therefore,
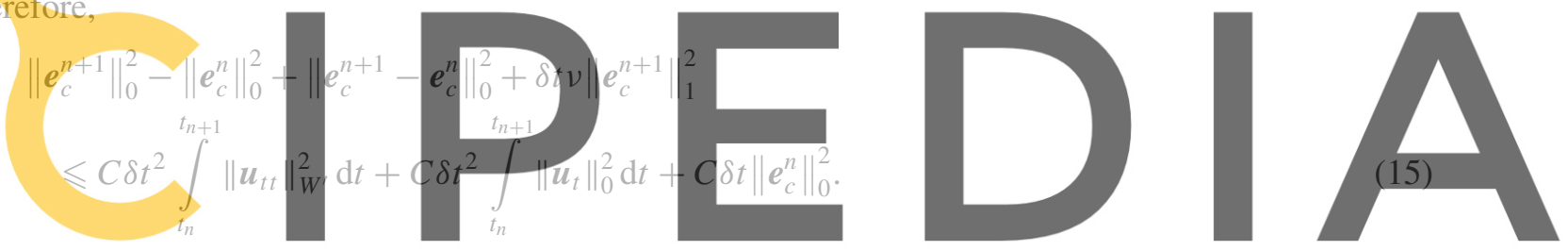

Adding up (15) for $n=0, \ldots, N$, and using the regularity properties (R2) and (R4) of the continuous

egister forlffiede ute

$$
\left\|e_{c}^{N+1}\right\|_{0}^{2}+\sum_{n=0}^{N}\left\|e_{c}^{n+1}-e_{c}^{n}\right\|_{0}^{2}+\delta t \nu \sum_{n=0}^{N}\left\|e_{c}^{n+1}\right\|_{1}^{2} \leqslant C \delta t^{2}+C \delta t \sum_{n=0}^{N}\left\|e_{c}^{n}\right\|_{0}^{2} .
$$

Applying the discrete Gronwall inequality, this implies

$$
\left\|\boldsymbol{e}_{c}^{N+1}\right\|_{0}^{2}+\sum_{n=0}^{N}\left\|\boldsymbol{e}_{c}^{n+1}-\boldsymbol{e}_{c}^{n}\right\|_{0}^{2}+\delta t v \sum_{n=0}^{N}\left\|\boldsymbol{e}_{c}^{n+1}\right\|_{1}^{2} \leqslant C \delta t^{2}
$$

and (11) follows.

Nonlinear case. When $\varepsilon=1$ we have

$$
\begin{aligned}
2 \delta t \mathrm{NLT} & =2 \delta t\left(c\left(\boldsymbol{u}^{n+1}, \boldsymbol{u}^{n+1}, \boldsymbol{e}_{c}^{n+1}\right)-c\left(\boldsymbol{u}\left(t_{n+1}\right), \boldsymbol{u}\left(t_{n+1}\right), \boldsymbol{e}_{c}^{n+1}\right)\right) \\
& =2 \delta t\left(-c\left(\boldsymbol{u}^{n+1}, \boldsymbol{e}_{c}^{n+1}, \boldsymbol{e}_{c}^{n+1}\right)-c\left(\boldsymbol{e}_{c}^{n+1}, \boldsymbol{u}\left(t_{n+1}\right), \boldsymbol{e}_{c}^{n+1}\right)\right) \\
& =T_{1}+T_{2},
\end{aligned}
$$

where again $T_{1}=0$ due to the skew-symmetry of the trilinear form $c$, and

$$
\begin{aligned}
T_{2} & =-2 \delta t c\left(\boldsymbol{e}_{c}^{n+1}, \boldsymbol{u}\left(t_{n+1}\right), \boldsymbol{e}_{c}^{n+1}\right) \\
& \leqslant C \delta t\left\|\boldsymbol{e}_{c}^{n+1}\right\|_{0}\left\|\boldsymbol{u}\left(t_{n+1}\right)\right\|_{2}\left\|\boldsymbol{e}_{c}^{n+1}\right\|_{1} \leqslant \frac{\delta t v}{3}\left\|\nabla \boldsymbol{e}_{c}^{n+1}\right\|_{0}^{2}+C \delta t\left\|\boldsymbol{e}_{c}^{n+1}\right\|_{0}^{2} .
\end{aligned}
$$


Therefore,

$$
\begin{aligned}
& \left\|\boldsymbol{e}_{c}^{n+1}\right\|_{0}^{2}-\left\|\boldsymbol{e}_{c}^{n}\right\|_{0}^{2}+\left\|\boldsymbol{e}_{c}^{n+1}-\boldsymbol{e}_{c}^{n}\right\|_{0}^{2}+\delta t v\left\|\boldsymbol{e}_{c}^{n+1}\right\|_{1}^{2} \\
& \quad \leqslant C \delta t^{2} \int_{t_{n}}^{t_{n+1}}\left\|\boldsymbol{u}_{t t}\right\|_{W^{\prime}}^{2} \mathrm{~d} t+C \delta t^{2} \int_{t_{n}}^{t_{n+1}}\left\|\boldsymbol{u}_{t}\right\|_{0}^{2} \mathrm{~d} t+C \delta t\left\|\boldsymbol{e}_{c}^{n+1}\right\|_{0}^{2}
\end{aligned}
$$

and

$$
\left\|\boldsymbol{e}_{c}^{N+1}\right\|_{0}^{2}+\sum_{n=0}^{N}\left\|\boldsymbol{e}_{c}^{n+1}-\boldsymbol{e}_{c}^{n}\right\|_{0}^{2}+\delta t \nu \sum_{n=0}^{N}\left\|\boldsymbol{e}_{c}^{n+1}\right\|_{1}^{2} \leqslant C \delta t^{2}+C \delta t \sum_{n=0}^{N}\left\|\boldsymbol{e}_{c}^{n+1}\right\|_{0}^{2} .
$$

Applying the discrete Gronwall inequality, this implies, for sufficiently small $\delta t$,

$$
\left\|\boldsymbol{e}_{c}^{N+1}\right\|_{0}^{2}+\sum_{n=0}^{N}\left\|\boldsymbol{e}_{c}^{n+1}-\boldsymbol{e}_{c}^{n}\right\|_{0}^{2}+\delta t v \sum_{n=0}^{N}\left\|\boldsymbol{e}_{c}^{n+1}\right\|_{1}^{2} \leqslant C \delta t^{2}
$$

and (11) follows again.

Remark 1. The error estimates proved in Theorem 1 ensure that the semidiscrete velocit

first order accurate in the

$l^{s}(X)$ denote the space of
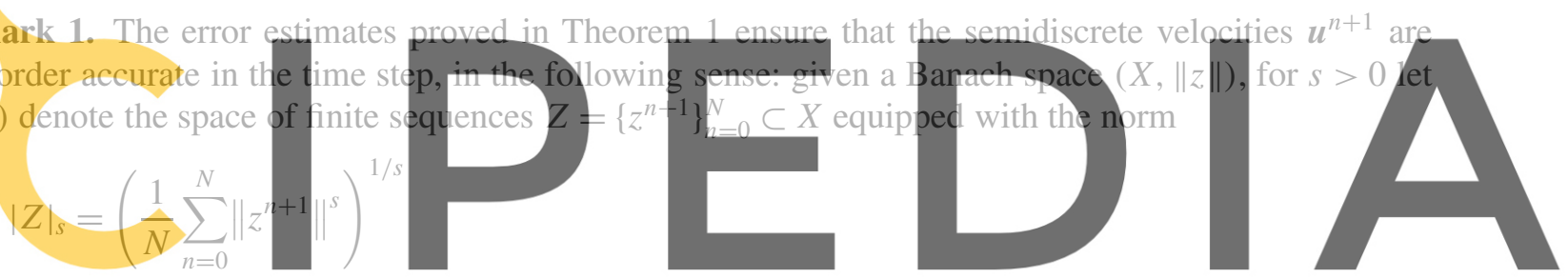

for $s<\infty$ and $|Z|_{\infty}=\max _{n=0, \ldots N}\left\|z^{n+1}\right\|$. Then, $\boldsymbol{u}^{n+1}$ is first order accurate in $l^{\infty}\left(\boldsymbol{L}^{2}(\Omega)\right)$ and in

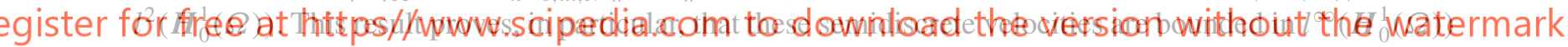

by a constant independent of $\delta t$, since:

$$
\left\|\boldsymbol{u}^{n+1}\right\|_{1} \leqslant\left\|\boldsymbol{u}\left(\hat{t}_{n+1}\right)\right\|_{1}+\left\|\boldsymbol{e}_{c}^{n+1}\right\|_{1} \leqslant\left\|\boldsymbol{u}\left(t_{n+1}\right)\right\|_{1}+(C \delta \boldsymbol{t})^{1 / 2} \leqslant C
$$

due to Theorem 1 and the regularity assumed on the continuous solution. Moreover, we also have $\left\|\boldsymbol{e}_{c}^{n+1}\right\|_{1} \leqslant C \delta t^{1 / 2}$. We will use these results later on.

We also have an error estimate for the semidiscrete pressure $p^{n+1}$ :

Proposition 1. Let (R1)-(R4) hold. Then, there is a constant $C$ independent of $\delta t$ such that

$$
\delta t \sum_{n=0}^{N}\left\|r_{c}^{n+1}\right\|_{0}^{2} \leqslant C \delta t
$$

If $\varepsilon=1$, (19) holds for sufficiently small $\delta t$.

Proof. By the continuous inf-sup condition (5), we have, using (13),

$$
\begin{aligned}
\left\|r_{c}^{n+1}\right\|_{0} & \leqslant C \sup _{\boldsymbol{v} \in V} \frac{\left(\nabla r_{c}^{n+1}, \boldsymbol{v}\right)}{\|\boldsymbol{v}\|_{1}} \\
& =C \sup _{\boldsymbol{v} \in V} \frac{1}{\|\boldsymbol{v}\|_{1}}\left\{\frac{-1}{\delta t}\left(\boldsymbol{e}_{c}^{n+1}-\boldsymbol{e}_{c}^{n}, \boldsymbol{v}\right)-v\left(\nabla \boldsymbol{e}_{c}^{n+1}, \nabla \boldsymbol{v}\right)+\left\langle\boldsymbol{R}^{n}, \boldsymbol{v}\right\rangle\right\}+\text { NLT. }
\end{aligned}
$$


We bound each term as follows (for the Taylor residual term, see [21]):

$$
\begin{aligned}
& \frac{1}{\delta t\|\boldsymbol{v}\|_{1}}\left(\boldsymbol{e}_{c}^{n+1}-\boldsymbol{e}_{c}^{n}, \boldsymbol{v}\right) \leqslant \frac{C}{\delta t}\left\|\boldsymbol{e}_{c}^{n+1}-\boldsymbol{e}_{c}^{n}\right\|_{0}, \\
& \frac{v}{\|\boldsymbol{v}\|_{1}}\left(\nabla \boldsymbol{e}_{c}^{n+1}, \nabla \boldsymbol{v}\right) \leqslant C v^{1 / 2}\left\|\boldsymbol{e}_{c}^{n+1}\right\|_{1}, \\
& \frac{1}{\|\boldsymbol{v}\|_{1}}\left\langle\boldsymbol{R}^{n}, \boldsymbol{v}\right\rangle \leqslant\left\|\boldsymbol{R}^{n}\right\|_{-1} \leqslant C\left(\int_{t_{n}}^{t_{n+1}} t\left\|\boldsymbol{u}_{t t}\right\|_{-1}^{2} \mathrm{~d} t\right)^{1 / 2} .
\end{aligned}
$$

The treatment of the NLT is again different for $\varepsilon=0$ and 1 .

Linearized case. Using the continuity properties of the trilinear form $c$, the regularity property (R1) of $\boldsymbol{u}$ and the results of Theorem 1 and Remark 1, we have

$$
\begin{aligned}
& \mathrm{NLT}=\frac{1}{\|\boldsymbol{v}\|_{1}}\left(c\left(\boldsymbol{u}^{n}, \boldsymbol{u}^{n+1}, \boldsymbol{v}\right)-c\left(\boldsymbol{u}\left(t_{n+1}\right), \boldsymbol{u}\left(t_{n+1}\right), \boldsymbol{v}\right)\right) \\
& =\frac{1}{\|v\|_{1}}\left(-c\left(\boldsymbol{u}^{n}, \boldsymbol{e}_{c}^{n+1}, v\right)-c\left(\boldsymbol{e}_{c}^{n}, \boldsymbol{u}\left(t_{n+1}\right), v\right)-c\left(\boldsymbol{u}\left(t_{n+1}\right)-\boldsymbol{u}\left(t_{n}\right), \boldsymbol{u}\left(t_{n+1}\right), v\right)\right) \\
& \begin{aligned}
& =T_{1}+T_{2}+T_{3}, \\
T_{1} & =\frac{-1}{\|v\|_{1}} c\left(\boldsymbol{u}^{n}, \boldsymbol{e}_{c}^{n+1}, v\right) \leqslant C\left\|u^{n}\right\|_{1}\left\|\boldsymbol{e}_{c}^{n+1}\right\|_{1} \leqslant C v^{1 / 2} \| \boldsymbol{e}_{c}^{n+1} \\
T_{2} & =\frac{-1}{\|v\|_{1}} c\left(\boldsymbol{e}_{c}^{n}, \boldsymbol{u}\left(t_{n+1}\right), \boldsymbol{v}\right) \leqslant C\left\|\boldsymbol{e}_{c}^{n}\right\|_{0}\left\|u\left(t_{n+1}\right)\right\|_{2} \leqslant C \mid \boldsymbol{e}_{c}^{n} \|_{0}
\end{aligned}
\end{aligned}
$$

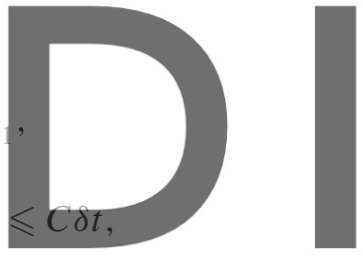

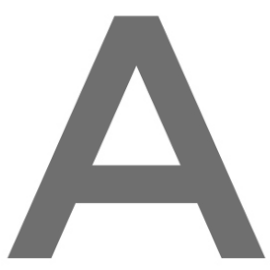

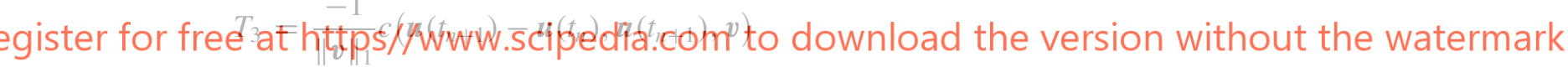

$$
\leqslant C\left\|\boldsymbol{u}\left(t_{n+1}\right)-\boldsymbol{u}\left(t_{n}\right)\right\|_{0}\left\|\boldsymbol{u}\left(t_{n+1}\right)\right\|_{2} \leqslant C \delta t^{1 / 2}\left(\int_{t_{n}}^{t_{n+1}}\left\|\boldsymbol{u}_{t}\right\|_{0}^{2} \mathrm{~d} t\right)^{1 / 2} .
$$

Therefore,

$$
\left\|r_{c}^{n+1}\right\|_{0}^{2} \leqslant C\left(\frac{1}{\delta t^{2}}\left\|\boldsymbol{e}_{c}^{n+1}-\boldsymbol{e}_{c}^{n}\right\|_{0}^{2}+v\left\|\boldsymbol{e}_{c}^{n+1}\right\|_{1}^{2}+\int_{t_{n}}^{t_{n+1}} t\left\|\boldsymbol{u}_{t t}\right\|_{-1}^{2} \mathrm{~d} t+\delta t^{2}+\delta t \int_{t_{n}}^{t_{n+1}}\left\|\boldsymbol{u}_{t}\right\|_{0}^{2} \mathrm{~d} t\right) .
$$

Finally,

$$
\begin{aligned}
\delta t \sum_{n=0}^{N}\left\|r_{c}^{n+1}\right\|_{0}^{2} \leqslant C( & \frac{1}{\delta t} \sum_{n=0}^{N}\left\|\boldsymbol{e}_{c}^{n+1}-\boldsymbol{e}_{c}^{n}\right\|_{0}^{2}+v \delta t \sum_{n=0}^{N}\left\|\boldsymbol{e}_{c}^{n+1}\right\|_{1}^{2} \\
& \left.+\delta t \int_{0}^{T} t\left\|\boldsymbol{u}_{t t}\right\|_{-1}^{2} \mathrm{~d} t+\delta t^{2}+\delta t^{2} \int_{0}^{T}\left\|\boldsymbol{u}_{t}\right\|_{0}^{2} \mathrm{~d} t\right) \\
\leqslant & \\
\leqslant & +C \delta t \int_{0}^{T} t\left\|\boldsymbol{u}_{t t}\right\|_{-1}^{2} \mathrm{~d} t+C \delta t^{2} \int_{0}^{T}\left\|\boldsymbol{u}_{t}\right\|_{0}^{2} \mathrm{~d} t
\end{aligned}
$$


due to (16). Estimate (19) follows from the regularity properties (R2) and (R3) of $\boldsymbol{u}$.

Nonlinear case. This time we have:

$$
\begin{aligned}
\mathrm{NLT} & =\frac{1}{\|\boldsymbol{v}\|_{1}}\left(c\left(\boldsymbol{u}^{n+1}, \boldsymbol{u}^{n+1}, \boldsymbol{v}\right)-c\left(\boldsymbol{u}\left(t_{n+1}\right), \boldsymbol{u}\left(t_{n+1}\right), \boldsymbol{v}\right)\right) \\
& =\frac{1}{\|\boldsymbol{v}\|_{1}}\left(-c\left(\boldsymbol{u}^{n+1}, \boldsymbol{e}_{c}^{n+1}, \boldsymbol{v}\right)-c\left(\boldsymbol{e}_{c}^{n+1}, \boldsymbol{u}\left(t_{n+1}\right), \boldsymbol{v}\right)\right) \\
& =T_{1}+T_{2}, \\
T_{1} & =\frac{-1}{\|\boldsymbol{v}\|_{1}} c\left(\boldsymbol{u}^{n+1}, \boldsymbol{e}_{c}^{n+1}, \boldsymbol{v}\right) \leqslant C v^{1 / 2}\left\|\boldsymbol{e}_{c}^{n+1}\right\|_{1}, \\
T_{2} & =\frac{-1}{\|\boldsymbol{v}\|_{1}} c\left(\boldsymbol{e}_{c}^{n+1}, \boldsymbol{u}\left(t_{n+1}\right), \boldsymbol{v}\right) \leqslant C\left\|\boldsymbol{e}_{c}^{n+1}\right\|_{0} \leqslant C \delta t
\end{aligned}
$$

and (19) follows again.

\subsection{A priori stability estimate}

We begin the analysis of the discrete problem recalling a stability estimate which was proven in [7] under weak regularity assumptions on the continuous solution. When studying pressure-gradientprojection methods for steady, incompressible flow problems, the following assumptions are encountered (see [5]), all of which carry over to the unsteady case:

H1. There exist $\alpha_{-}>0$ and $\alpha_{+}>0$ independent of $h$ such that:

$$
\alpha_{-} h^{2} \leqslant \alpha \leqslant \alpha_{+} h^{2} \text {. }
$$

This assumption dictates the behaviour of the numerical parameter $\alpha$.

H2. The family of finite element partitions $\Theta_{h}$ is quasi-uniform, that is, there exists a constant $\sigma>0$ independent of $h$ such that, for all $h>0$ :

$$
\min \left\{\operatorname{diam}\left(B_{K}\right) \mid K \in \Theta_{h}\right\} \geqslant \sigma \max \left\{\operatorname{diam}\left(B_{K}\right) \mid K \in \Theta_{h}\right\},
$$

where $B_{K}$ is the largest ball contained in K. Condition (21) is needed in order to have the following inverse estimate (see [2]):

$$
\left\|\boldsymbol{v}_{h}\right\|_{1} \leqslant \frac{C}{h}\left\|\boldsymbol{v}_{h}\right\|_{0}, \quad \forall \boldsymbol{v}_{h} \in V_{h} .
$$

This assumption can be weakened by using local parameters $\alpha_{K}$ (see [6]).

H3. As in [5,6], let $\nabla Q_{h}$ denote the space

$$
\nabla Q_{h}=\left\{\boldsymbol{v}_{h} \in \boldsymbol{L}^{2}(\Omega) \mid \boldsymbol{v}_{h}=\nabla q_{h}, q_{h} \in Q_{h}\right\}
$$

and define the space $E_{h}$ by

$$
E_{h}=V_{h}+\nabla Q_{h} \subset \boldsymbol{L}^{2}(\Omega) .
$$


We consider three mutually orthogonal subspaces $E_{h, i}$ of $E_{h}$ defined by

$$
E_{h, 1}=V_{h, 0}, \quad E_{h, 2}=V_{h, 0}^{\perp} \cap V_{h}, \quad E_{h, 3}=V_{h}^{\perp} \cap E_{h}
$$

so that

$$
E_{h}=E_{h, 1} \oplus E_{h, 2} \oplus E_{h, 3} .
$$

For $i=1,2,3$, we call $P_{h, i}$ the $L^{2}$-projection of $E_{h}$ onto $E_{h, i}$, and for $i \neq j, P_{h, i j}=P_{h, i}+P_{h, j}$ and $E_{h, i j}=E_{h, i} \oplus E_{h, j}$. In this notation, $\xi_{h}^{n+1}=P_{h, 12}\left(\nabla p_{h}^{n+1}\right)$. We assume that there is a constant $\beta_{0}$ independent of $h$ such that

$$
\left\|\nabla q_{h}\right\|_{0} \leqslant \beta_{0}\left\|P_{h, 13}\left(\nabla q_{h}\right)\right\|_{0},
$$

that is to say, that the second component of the decomposition of every $\nabla q_{h}$ in $E_{h}$ can be bounded in terms of the other two. This condition can also be written in the form

$$
\inf _{q_{h} \in Q_{h}}\left(\sup _{\boldsymbol{v}_{h} \in E_{h, 13}} \frac{\left(\nabla q_{h}, \boldsymbol{v}_{h}\right)}{\left\|\boldsymbol{v}_{h}\right\|_{0}\left\|\nabla q_{h}\right\|_{0}}\right) \geqslant \beta_{1}>0,
$$

in a similar way to the classical inf-sup condition; condition (24), however, is weaker since the space where the supremum is taken, $E_{h, 13}$, is larger than in the classical case, $V_{h, 0}=E_{h, 1}$. Condition (24) was analyzed in [5], where it was shown to be satisfied by equal order simplicial finite element interpolations.

The scheme analyzed in [7] differs slightly from (8)-(10) in the interpretation of the parameter $\alpha$ and the pressure gradient projection $\xi_{h}^{n+1}$; moreover, it is restricted to the case $\varepsilon=1$. However, a straightforward extension of the proofs in [7] leads to the following stability result:

Theorem 2. Assume $\mathrm{H} 1-\mathrm{H} 3$ hold; then, there exists a constant $C>0$ independent of $\delta t$ and $h$ such that, for small enough $\delta t$ :

$$
\left\|\boldsymbol{u}_{h}^{N+1}\right\|_{0}^{2}+v \delta t \sum_{n=0}^{N}\left\|\boldsymbol{u}_{h}^{n+1}\right\|_{1}^{2}+\delta t h \sum_{n=0}^{N}\left\|\nabla p_{h}^{n+1}\right\|_{0} \leqslant C .
$$

Remark 2. This theorem proves that the discrete velocities are stable in $l^{\infty}\left(\boldsymbol{L}^{2}(\Omega)\right)$ and $l^{2}\left(\boldsymbol{H}_{0}^{1}(\Omega)\right)$, while the discrete pressure gradients (scaled by $h$ ) are stable in $l^{1}\left(\boldsymbol{L}^{2}(\Omega)\right)$; this proves, in particular, that the discrete problem is always well-posed. The result for the pressure can be improved to $l^{2}\left(\boldsymbol{L}^{2}(\Omega)\right)$ in 2D flows or for the linear Stokes case (see [7]). We improve this estimates later on to $l^{\infty}\left(\boldsymbol{H}_{0}^{1}(\Omega)\right)$ for the velocity and $l^{2}\left(\boldsymbol{L}^{2}(\Omega)\right)$ for the pressure as a consequence of the error estimates of the next section.

\subsection{Error estimates for the velocity}

We now proceed to obtain error estimates for the fully discrete velocity solution $\boldsymbol{u}_{h}^{n+1}$ as an approximation of the semidiscrete solution $\boldsymbol{u}^{n+1}$ under stronger regularity assumptions on the continuous problem. For simplicity, we assume that the domain $\Omega$ is polyhedral, so that it can be exactly covered by triangulations. We define and split the errors of the method as

$$
\begin{aligned}
& \boldsymbol{e}^{n+1}=\boldsymbol{u}\left(t_{n+1}\right)-\boldsymbol{u}_{h}^{n+1}=\boldsymbol{e}_{c}^{n+1}+\boldsymbol{e}_{d}^{n+1}, \\
& r^{n+1}=p\left(t_{n+1}\right)-p_{h}^{n+1}=r_{c}^{n+1}+r_{d}^{n+1}, \\
& \boldsymbol{g}^{n+1}=\nabla p\left(t_{n+1}\right)-\boldsymbol{\xi}_{h}^{n+1}=\boldsymbol{g}_{c}^{n+1}+\boldsymbol{g}_{d}^{n+1},
\end{aligned}
$$


where the discrete errors are defined as

$$
\boldsymbol{e}_{d}^{n+1}=\boldsymbol{u}^{n+1}-\boldsymbol{u}_{h}^{n+1}, \quad r_{d}^{n+1}=p^{n+1}-p_{h}^{n+1}, \quad \boldsymbol{g}_{d}^{n+1}=\nabla p^{n+1}-\boldsymbol{\xi}_{h}^{n+1} .
$$

Subtracting (8) (with $\theta=1$ ) from (6) and (9) (with $\beta=1$ ) from (7), it can be seen that these discrete errors satisfy the following equations, which hold for any $\left(\boldsymbol{v}_{h}, q_{h}, \boldsymbol{\eta}_{h}\right) \in V_{h, 0} \times Q_{h} \times V_{h}$ :

$$
\begin{aligned}
& \left(\frac{\boldsymbol{e}_{d}^{n+1}-\boldsymbol{e}_{d}^{n}}{\delta t}, \boldsymbol{v}_{h}\right)+a\left(\boldsymbol{e}_{d}^{n+1}, \boldsymbol{v}_{h}\right)+\left(\nabla r_{d}^{n+1}, \boldsymbol{v}_{h}\right)-c\left(\boldsymbol{u}_{h}^{n+\varepsilon}, \boldsymbol{u}_{h}^{n+1}, \boldsymbol{v}_{h}\right)+c\left(\boldsymbol{u}^{n+\varepsilon}, \boldsymbol{u}^{n+1}, \boldsymbol{v}_{h}\right)=0, \\
& \left(\nabla \cdot \boldsymbol{e}_{d}^{n+1}, q_{h}\right)+\alpha\left(\left(\nabla r_{d}^{n+1}, \nabla q_{h}\right)-\left(\boldsymbol{g}_{d}^{n+1}, \nabla q_{h}\right)\right)=0 \\
& -\left(\nabla r_{d}^{n+1}, \boldsymbol{\eta}_{h}\right)+\left(\boldsymbol{g}_{d}^{n+1}, \boldsymbol{\eta}_{h}\right)=0 .
\end{aligned}
$$

We also introduce the following notation. Given $\left(\boldsymbol{v}_{h}, q_{h}, \boldsymbol{\eta}_{h}\right) \in V_{h, 0} \times Q_{h} \times V_{h}$ arbitrary, we call:

$$
\begin{array}{ll}
I_{0}\left(\boldsymbol{u}^{n+1}, \boldsymbol{v}_{h}\right)=\left\|\boldsymbol{u}^{n+1}-\boldsymbol{v}_{h}\right\|_{0}, & I_{1}\left(\boldsymbol{u}^{n+1}, \boldsymbol{v}_{h}\right)=\left\|\boldsymbol{u}^{n+1}-\boldsymbol{v}_{h}\right\|_{1}, \\
I_{0}\left(p^{n+1}, q_{h}\right)=\left\|p^{n+1}-q_{h}\right\|_{0}, & I_{1}\left(p^{n+1}, q_{h}\right)=\left\|\nabla p^{n+1}-\nabla q_{h}\right\|_{0}, \\
I_{0}\left(\nabla p^{n+1}, \boldsymbol{\eta}_{h}\right)=\left\|\nabla p^{n+1}-\boldsymbol{\eta}_{h}\right\|_{0}, & G_{n+1}=\left\|\boldsymbol{\xi}_{h}^{n+1}-\nabla p_{h}^{n+1}\right\|_{0}
\end{array}
$$

and

$$
\begin{aligned}
E_{n}(h)= & \inf _{\boldsymbol{v}_{h} \in V_{h, 0}}\left\|\boldsymbol{u}^{n+1}-\boldsymbol{v}_{h}\right\|_{1}+\frac{1}{h} \inf _{\boldsymbol{v}_{h} \in V_{h, 0}}\left\|\boldsymbol{u}^{n+1}-\boldsymbol{v}_{h}\right\|_{0}+\inf _{q_{h} \in Q_{h}}\left\|p^{n+1}-q_{h}\right\|_{0} \\
& +h \inf _{q_{h} \in Q_{h}}\left\|\nabla p^{n+1}-\nabla q_{h}\right\|_{0}+h \inf _{\eta_{h} \in V_{h}}\left\|\nabla p^{n+1}-\boldsymbol{\eta}_{h}\right\|_{0}, \\
E(h)= & \max _{n=0, \ldots, N} E_{n}(h) .
\end{aligned}
$$

We begin with a rather technical lemma:

Lemma 1. Assume $\mathrm{H} 2$ and $\mathrm{H} 3$ hold; then, for $n=0, \ldots, N$, for any $\left(\boldsymbol{v}_{h}, q_{h}, \boldsymbol{\eta}_{h}\right) \in V_{h, 0} \times Q_{h} \times V_{h}$ and for small enough $h$ :

$$
\begin{aligned}
\left\|\nabla r_{d}^{n+1}\right\|_{0} \leqslant C\{ & I_{0}\left(\nabla p^{n+1}, \boldsymbol{\eta}_{h}\right)+I_{1}\left(p^{n+1}, q_{h}\right)+\frac{1}{\delta t}\left\|\boldsymbol{e}_{d}^{n+1}-\boldsymbol{e}_{d}^{n}\right\|_{0} \\
& +G_{n+1}+\frac{v^{1 / 2}}{h}\left(\left\|\boldsymbol{e}_{d}^{n+1}\right\|_{1}+\left\|\boldsymbol{e}_{d}^{n}\right\|_{1}\right) \\
& \left.+\frac{v}{h}\left(\left\|\boldsymbol{e}_{d}^{n+1}\right\|_{1}^{2}+\left\|\boldsymbol{e}_{d}^{n}\right\|_{1}^{2}+\left\|\boldsymbol{e}_{c}^{n+1}\right\|_{1}^{2}+\left\|\boldsymbol{e}_{c}^{n}\right\|_{1}^{2}\right)\right\} .
\end{aligned}
$$

Proof. By the triangle inequality and the previous definitions, we have

$$
\begin{aligned}
\left\|\nabla r_{c}^{n+1}\right\|_{0} \leqslant & \left\|\nabla p^{n+1}-P_{h, 12}\left(\nabla q_{h}\right)\right\|_{0}+\left\|P_{h, 1}\left(\nabla q_{h}\right)-P_{h, 1}\left(\nabla p_{h}^{n+1}\right)\right\|_{0} \\
& +\left\|P_{h, 2}\left(\nabla q_{h}\right)-P_{h, 2}\left(\nabla p_{h}^{n+1}\right)\right\|_{0}+\left\|P_{h, 3}\left(\nabla p_{h}^{n+1}\right)\right\|_{0} \\
= & T_{1}+T_{2}+T_{3}+T_{4} .
\end{aligned}
$$

We bound each term separately. For the first term, we use a similar argument to that of [5] for the corresponding term in the analysis of an approximation of the Stokes problem, to get

$$
T_{1}=\left\|\nabla p^{n+1}-P_{h, 12}\left(\nabla q_{h}\right)\right\|_{0} \leqslant I_{0}\left(\nabla p^{n+1}, \boldsymbol{\eta}_{h}\right)+I_{1}\left(p^{n+1}, q_{h}\right) .
$$


For the second term, we have, due to the orthogonality of the projection $P_{h, 1}$,

$$
\begin{aligned}
T_{2}^{2} & =\left\|P_{h, 1}\left(\nabla q_{h}\right)-P_{h, 1}\left(\nabla p_{h}^{n+1}\right)\right\|_{0}^{2} \\
& =\left(P_{h, 1}\left(\nabla q_{h}-\nabla p_{h}^{n+1}\right), P_{h, 1}\left(\nabla q_{h}-\nabla p_{h}^{n+1}\right)\right) \\
& =\left(\nabla q_{h}-\nabla p^{n+1}, P_{h, 1}\left(\nabla q_{h}-\nabla p_{h}^{n+1}\right)\right)+\left(\nabla r_{d}^{n+1}, P_{h, 1}\left(\nabla q_{h}-\nabla p_{h}^{n+1}\right)\right) \\
& =T_{2, a}+T_{2, b}
\end{aligned}
$$

so that

$$
T_{2, a} \leqslant I_{1}\left(p^{n+1}, q_{h}\right)\left\|P_{h, 1}\left(\nabla q_{h}-\nabla p_{h}^{n+1}\right)\right\|_{0} .
$$

Moreover, taking $\boldsymbol{v}_{h}=P_{h, 1}\left(\nabla q_{h}-\nabla p_{h}^{n+1}\right) \in V_{h, 0}$ in (26), we get

$$
\begin{aligned}
T_{2, b}= & -\left(\frac{\boldsymbol{e}_{d}^{n+1}-\boldsymbol{e}_{d}^{n}}{\delta t}, P_{h, 1}\left(\nabla q_{h}-\nabla p_{h}^{n+1}\right)\right)-v\left(\nabla \boldsymbol{e}_{d}^{n+1}, \nabla P_{h, 1}\left(\nabla q_{h}-\nabla p_{h}^{n+1}\right)\right) \\
& +c\left(\boldsymbol{u}_{h}^{n+\varepsilon}, \boldsymbol{u}_{h}^{n+1}, P_{h, 1}\left(\nabla q_{h}-\nabla p_{h}^{n+1}\right)\right)-c\left(\boldsymbol{u}^{n+\varepsilon}, \boldsymbol{u}^{n+1}, P_{h, 1}\left(\nabla q_{h}-\nabla p_{h}^{n+1}\right)\right) \\
= & -\left(\frac{\boldsymbol{e}_{d}^{n+1}-\boldsymbol{e}_{d}^{n}}{\delta t}, P_{h, 1}\left(\nabla q_{h}-\nabla p_{h}^{n+1}\right)\right)-v\left(\nabla \boldsymbol{e}_{d}^{n+1}, \nabla P_{h, 1}\left(\nabla q_{h}-\nabla p_{h}^{n+1}\right)\right) \\
& -c\left(\boldsymbol{e}_{d}^{n+\varepsilon}, \boldsymbol{u}^{n+1}, P_{h, 1}\left(\nabla q_{h}-\nabla p_{h}^{n+1}\right)\right)-c\left(\boldsymbol{u}_{h}^{n+\varepsilon}, \boldsymbol{e}_{d}^{n+1}, P_{h, 1}\left(\nabla q_{h}-\nabla p_{h}^{n+1}\right)\right) .
\end{aligned}
$$

Then,

$$
\begin{aligned}
-\left(\frac{\boldsymbol{e}_{d}^{n+1}-\boldsymbol{e}_{d}^{n}}{\delta t}, P_{h, 1}\left(\nabla q_{h}-\nabla p_{h}^{n+1}\right)\right) & \leqslant \frac{1}{\delta t}\left\|\boldsymbol{e}_{d}^{n+1}-\boldsymbol{e}_{d}^{n}\right\|_{0}\left\|P_{h, 1}\left(\nabla q_{h}-\nabla p_{h}^{n+1}\right)\right\|_{0}, \\
-v\left(\nabla \boldsymbol{e}_{d}^{n+1}, \nabla P_{h, 1}\left(\nabla q_{h}-\nabla p_{h}^{n+1}\right)\right) & \leqslant C v^{1 / 2}\left\|\boldsymbol{e}_{d}^{n+1}\right\|_{1}\left\|P_{h, 1}\left(\nabla q_{h}-\nabla p_{h}^{n+1}\right)\right\|_{1} \\
& \leqslant C \frac{v^{1 / 2}}{h}\left\|\boldsymbol{e}_{d}^{n+1}\right\|_{1}\left\|P_{h, 1}\left(\nabla q_{h}-\nabla p_{h}^{n+1}\right)\right\|_{0}, \\
-c\left(\boldsymbol{e}_{d}^{n+\varepsilon}, \boldsymbol{u}^{n+1}, P_{h, 1}\left(\nabla q_{h}-\nabla p_{h}^{n+1}\right)\right) & \leqslant C\left\|\boldsymbol{e}_{d}^{n+\varepsilon}\right\|_{1}\left\|\boldsymbol{u}^{n+1}\right\|_{1}\left\|P_{h, 1}\left(\nabla q_{h}-\nabla p_{h}^{n+1}\right)\right\|_{1} \\
& \leqslant C \frac{v^{1 / 2}}{h}\left\|\boldsymbol{e}_{d}^{n+\varepsilon}\right\|_{1}\left\|P_{h, 1}\left(\nabla q_{h}-\nabla p_{h}^{n+1}\right)\right\|_{0}, \\
-c\left(\boldsymbol{u}_{h}^{n+\varepsilon}, \boldsymbol{e}_{d}^{n+1}, P_{h, 1}\left(\nabla q_{h}-\nabla p_{h}^{n+1}\right)\right) & \leqslant\left(\boldsymbol{e}_{d}^{n+\varepsilon}, \boldsymbol{e}_{d}^{n+1}, P_{h, 1}\left(\nabla q_{h}-\nabla p_{h}^{n+1}\right)\right)+c\left(\boldsymbol{e}_{c}^{n+\varepsilon}, \boldsymbol{e}_{d}^{n+1}, P_{h, 1}\left(\nabla q_{h}-\nabla p_{h}^{n+1}\right)\right) \\
-c\left(\boldsymbol{u}\left(t_{n+\varepsilon}\right), \boldsymbol{e}_{d}^{n+1}, P_{h, 1}\left(\nabla q_{h}-\nabla p_{h}^{n+1}\right)\right), & \leqslant C \frac{\nu}{h}\left\|\boldsymbol{e}_{d}^{n+\varepsilon}\right\|_{1}\left\|\boldsymbol{e}_{d}^{n+1}\right\|_{1}\left\|P_{h, 1}\left(\nabla q_{h}-\nabla p_{h}^{n+1}\right)\right\|_{0} \\
c\left(\boldsymbol{e}_{d}^{n+\varepsilon}, \boldsymbol{e}_{d}^{n+1}, P_{h, 1}\left(\nabla q_{h}-\nabla p_{h}^{n+1}\right)\right) & \leqslant C \boldsymbol{e}_{d}^{n+\varepsilon}\left\|_{1}\right\| \boldsymbol{e}_{d}^{n+1}\left\|_{1}\right\| P_{h, 1}\left(\nabla q_{h}-\nabla p_{h}^{n+1}\right) \|_{1} \\
& \leqslant C \frac{v}{h}\left(\left\|\boldsymbol{e}_{d}^{n+\varepsilon}\right\|_{1}^{2}+\left\|\boldsymbol{e}_{d}^{n+1}\right\|_{1}^{2}\right)\left\|P_{h, 1}\left(\nabla q_{h}-\nabla p_{h}^{n+1}\right)\right\|_{0}, \\
c\left(\boldsymbol{e}_{c}^{n+\varepsilon}, \boldsymbol{e}_{d}^{n+1}, P_{h, 1}\left(\nabla q_{h}-\nabla p_{h}^{n+1}\right)\right) & \leqslant C\left\|\boldsymbol{e}_{c}^{n+\varepsilon}\right\|_{1}\left\|\boldsymbol{e}_{d}^{n+1}\right\|_{1}\left\|P_{h, 1}\left(\nabla q_{h}-\nabla p_{h}^{n+1}\right)\right\|_{1} \\
& \leqslant C \frac{v}{h}\left\|\boldsymbol{e}_{c}^{n+\varepsilon}\right\|_{1}\left\|\boldsymbol{e}_{d}^{n+1}\right\|_{1}\left\|P_{h, 1}\left(\nabla q_{h}-\nabla p_{h}^{n+1}\right)\right\|_{0} \\
& \leqslant C \frac{v}{h}\left(\left\|\boldsymbol{e}_{c}^{n+\varepsilon}\right\|_{1}^{2}+\left\|\boldsymbol{e}_{d}^{n+1}\right\|_{1}^{2}\right)\left\|P_{h, 1}\left(\nabla q_{h}-\nabla p_{h}^{n+1}\right)\right\|_{0},
\end{aligned}
$$




$$
\begin{aligned}
-c\left(\boldsymbol{u}\left(t_{n+\varepsilon}\right), \boldsymbol{e}_{d}^{n+1}, P_{h, 1}\left(\nabla q_{h}-\nabla p_{h}^{n+1}\right)\right) & \leqslant C\left\|\boldsymbol{u}\left(t_{n+\varepsilon}\right)\right\|_{2}\left\|\boldsymbol{e}_{d}^{n+1}\right\|_{1}\left\|P_{h, 1}\left(\nabla q_{h}-\nabla p_{h}^{n+1}\right)\right\|_{0} \\
& \leqslant C\left\|\boldsymbol{e}_{d}^{n+1}\right\|_{1}\left\|P_{h, 1}\left(\nabla q_{h}-\nabla p_{h}^{n+1}\right)\right\|_{0}
\end{aligned}
$$

due to Remark 1 and the regularity of the continuous velocity. Assuming $h \leqslant C v^{1 / 2}$ in the last term, we get

$$
\begin{aligned}
T_{2} \leqslant C & \left(I_{1}\left(p^{n+1}, q_{h}\right)+\frac{1}{\delta t}\left\|\boldsymbol{e}_{d}^{n+1}-\boldsymbol{e}_{d}^{n}\right\|_{0}+\frac{v^{1 / 2}}{h}\left(\left\|\boldsymbol{e}_{d}^{n+1}\right\|_{1}+\left\|\boldsymbol{e}_{d}^{n}\right\|_{1}\right)\right. \\
& \left.+\frac{v}{h}\left(\left\|\boldsymbol{e}_{d}^{n+1}\right\|_{1}^{2}+\left\|\boldsymbol{e}_{d}^{n}\right\|_{1}^{2}+\left\|\boldsymbol{e}_{c}^{n+1}\right\|_{1}^{2}+\left\|\boldsymbol{e}_{c}^{n}\right\|_{1}\right)\right) .
\end{aligned}
$$

Moreover, due to condition (23) and since $P_{h, 3}=I d-P_{h, 12}$ and $\xi_{h}^{n+1}=P_{h, 12}\left(\nabla p_{h}^{n+1}\right.$ ), we have

$$
\begin{aligned}
T_{3} & =\left\|P_{h, 2}\left(\nabla q_{h}\right)-P_{h, 2}\left(\nabla p_{h}^{n+1}\right)\right\|_{0} \\
& \leqslant C\left(\left\|P_{h, 1}\left(\nabla q_{h}\right)-P_{h, 1}\left(\nabla p_{h}^{n+1}\right)\right\|_{0}+\left\|P_{h, 3}\left(\nabla q_{h}\right)-P_{h, 3}\left(\nabla p_{h}^{n+1}\right)\right\|_{0}\right) \\
& \leqslant C\left(T_{2}+\left\|P_{h, 3}\left(\nabla q_{h}\right)\right\|_{0}+\left\|P_{h, 3}\left(\nabla p_{h}^{n+1}\right)\right\|_{0}\right) \\
& \leqslant C\left(T_{2}+\left\|\nabla q_{h}-\nabla p^{n+1}\right\|_{0}+\left\|\nabla p^{n+1}-P_{h, 12}\left(\nabla q_{h}\right)\right\|_{0}+G_{n+1}\right) \\
& =C\left(T_{2}+I_{1}\left(p^{n+1}, q_{h}\right)+T_{1}+G_{n+1}\right) .
\end{aligned}
$$

Finally,

$$
T_{4}=\left\|P_{h, 3}\left(\nabla p_{h}^{n+1}\right)\right\|_{0}=G_{n+1}
$$

and (30) follows.

In our convergence analysis we will also need the following assumption:

H4. There exists $C>0$ independent of $h$ and $\delta t$ such that:

$$
\delta t \geqslant C h^{2} \text {. }
$$

This condition does not impose an upper bound on the time step, so that the method remains unconditionally stable (see also Remark 5). Our main result of this section is the following:

Theorem 3. Assume (R1), (R2), (R4) and $\mathrm{H} 1-\mathrm{H} 4$ hold; then, there exists a constant $C>0$ independent of $\delta t$ and $h$ such that, for small enough $h$ and, if $\varepsilon=1$, small enough $\delta t$,

$$
\left\|\boldsymbol{e}_{d}^{N+1}\right\|_{0}^{2}+v \delta t \sum_{n=0}^{N}\left\|\boldsymbol{e}_{d}^{n+1}\right\|_{1}^{2} \leqslant C\left((E(h))^{2}+E(h) \delta t^{2}\right) .
$$

Proof. Let us call

$$
\begin{aligned}
A= & \left(\frac{\boldsymbol{e}_{d}^{n+1}-\boldsymbol{e}_{d}^{n}}{\delta t}, \boldsymbol{e}_{d}^{n+1}\right)+v\left(\nabla \boldsymbol{e}_{d}^{n+1}, \nabla \boldsymbol{e}_{d}^{n+1}\right)+\left(\nabla r_{d}^{n+1}, \boldsymbol{e}_{d}^{n+1}\right)+\left(\nabla \cdot \boldsymbol{e}_{d}^{n+1}, r_{d}^{n+1}\right) \\
& +\alpha\left(\nabla r_{d}^{n+1}, \nabla r_{d}^{n+1}\right)-\alpha\left(\boldsymbol{g}_{d}^{n+1}, \nabla r_{d}^{n+1}\right)-\alpha\left(\boldsymbol{g}_{d}^{n+1}, \nabla r_{d}^{n+1}\right)+\alpha\left(\boldsymbol{g}_{d}^{n+1}, \boldsymbol{g}_{d}^{n+1}\right) \\
= & \frac{1}{2 \delta t}\left(\left\|\boldsymbol{e}_{d}^{n+1}\right\|_{0}^{2}-\left\|\boldsymbol{e}_{d}^{n}\right\|_{0}^{2}+\left\|\boldsymbol{e}_{d}^{n+1}-\boldsymbol{e}_{d}^{n}\right\|_{0}^{2}\right)+v\left\|\boldsymbol{e}_{d}^{n+1}\right\|_{1}^{2}+\alpha\left\|\boldsymbol{\xi}_{h}^{n+1}-\nabla p_{h}^{n+1}\right\|_{0}^{2} .
\end{aligned}
$$


Given $\left(\boldsymbol{v}_{h}, q_{h}, \boldsymbol{\eta}_{h}\right) \in V_{h, 0} \times Q_{h} \times V_{h}$ arbitrary, we take $\boldsymbol{v}_{h}-\boldsymbol{u}_{h}^{n+1}, q_{h}-p_{h}^{n+1}$ and $\boldsymbol{\eta}_{h}-\boldsymbol{\xi}_{h}^{n+1}$ as test functions in (26), (27) and (28), respectively, to get

$$
\begin{aligned}
A= & \left(\frac{\boldsymbol{e}_{d}^{n+1}-\boldsymbol{e}_{d}^{n}}{\delta t}, \boldsymbol{u}^{n+1}-\boldsymbol{v}_{h}\right)+v\left(\nabla \boldsymbol{e}_{d}^{n+1}, \nabla\left(\boldsymbol{u}^{n+1}-\boldsymbol{v}_{h}\right)\right) \\
& +\left(\nabla r_{d}^{n+1}, \boldsymbol{u}^{n+1}-\boldsymbol{v}_{h}\right)+c\left(\boldsymbol{u}_{h}^{n+\varepsilon}, \boldsymbol{u}_{h}^{n+1}, \boldsymbol{u}_{h}^{n+1}-\boldsymbol{v}_{h}\right)-c\left(\boldsymbol{u}^{n+\varepsilon}, \boldsymbol{u}^{n+1}, \boldsymbol{u}_{h}^{n+1}-\boldsymbol{v}_{h}\right) \\
& +\left(\nabla \cdot \boldsymbol{e}_{d}^{n+1}, p^{n+1}-q_{h}\right)+\alpha\left(\nabla r_{d}^{n+1}-\boldsymbol{g}_{d}^{n+1}, \boldsymbol{\eta}_{h}-\nabla q_{h}\right) .
\end{aligned}
$$

We bound each term as follows:

$$
\begin{aligned}
& \left(\frac{\boldsymbol{e}_{d}^{n+1}-\boldsymbol{e}_{d}^{n}}{\delta t}, \boldsymbol{u}^{n+1}-\boldsymbol{v}_{h}\right) \leqslant \frac{1}{\delta t}\left\|\boldsymbol{e}_{d}^{n+1}-\boldsymbol{e}_{d}^{n}\right\|_{0} I_{0}\left(\boldsymbol{u}^{n+1}, \boldsymbol{v}_{h}\right) \leqslant \frac{1}{4 \delta t}\left\|\boldsymbol{e}_{d}^{n+1}-\boldsymbol{e}_{d}^{n}\right\|_{0}^{2}+\frac{C}{\delta t} I_{0}\left(\boldsymbol{u}^{n+1}, \boldsymbol{v}_{h}\right)^{2}, \\
& v\left(\nabla \boldsymbol{e}_{d}^{n+1}, \nabla\left(\boldsymbol{u}^{n+1}-\boldsymbol{v}_{h}\right)\right) \leqslant C v\left\|\boldsymbol{e}_{d}^{n+1}\right\|_{1} I_{1}\left(\boldsymbol{u}^{n+1}, \boldsymbol{v}_{h}\right) \leqslant \frac{v}{10}\left\|\boldsymbol{e}_{d}^{n+1}\right\|_{1}^{2}+C I_{1}\left(\boldsymbol{u}^{n+1}, \boldsymbol{v}_{h}\right)^{2}, \\
& \left(\nabla \cdot \boldsymbol{e}_{d}^{n+1}, p^{n+1}-q_{h}\right) \leqslant \frac{v}{10}\left\|\boldsymbol{e}_{d}^{n+1}\right\|_{1}^{2}+C I_{0}\left(p^{n+1}, q_{h}\right)^{2}, \\
& \begin{aligned}
\alpha\left(\nabla r_{d}^{n+1}-\boldsymbol{g}_{d}^{n+1}, \boldsymbol{\eta}_{h}-\nabla q_{h}\right) & =\alpha\left(\nabla p_{h}^{n+1}-\boldsymbol{\xi}_{h}^{n+1}, \boldsymbol{\eta}_{h}-\nabla q_{h}\right) \\
& \leqslant \alpha G_{n+1}\left(I_{0}\left(\nabla p^{n+1}, \boldsymbol{\eta}_{h}\right)+I_{1}\left(p^{n+1}, q_{h}\right)\right) \\
& \leqslant C h^{2} G_{n+1}\left(I_{0}\left(\nabla p^{n+1}, \boldsymbol{\eta}_{h}\right)+I_{1}\left(p^{n+1}, q_{h}\right)\right) \\
& \leqslant \frac{\alpha_{-} h^{2}}{3} G_{n+1}^{2}+C h^{2} I_{0}\left(\nabla p^{n+1}, \boldsymbol{\eta}_{h}\right)^{2}+C h^{2} I_{1}\left(p^{n+1}, q_{h}\right)^{2},
\end{aligned}
\end{aligned}
$$

where $\alpha_{-}$was defined in (20). Moreover, due to Lemma 1 we have

$$
\begin{aligned}
\left(\nabla r_{d}^{n+1}, \boldsymbol{u}^{n+1}-\boldsymbol{v}_{h}\right) \leqslant & \left\|\nabla r_{d}^{n+1}\right\|_{0} I_{0}\left(\boldsymbol{u}^{n+1}, \boldsymbol{v}_{h}\right) \\
\leqslant & C\left(I_{0}\left(\nabla p^{n+1}, \boldsymbol{\eta}_{h}\right)+I_{1}\left(p^{n+1}, q_{h}\right)\right. \\
& \quad+\frac{1}{\delta t}\left\|\boldsymbol{e}_{d}^{n+1}-\boldsymbol{e}_{d}^{n}\right\|_{0}+G_{n+1}+\frac{v^{1 / 2}}{h}\left(\left\|\boldsymbol{e}_{d}^{n+1}\right\|_{1}+\left\|\boldsymbol{e}_{d}^{n}\right\|_{1}\right) \\
& \left.\quad+\frac{v}{h}\left(\left\|\boldsymbol{e}_{d}^{n+1}\right\|_{1}^{2}+\left\|\boldsymbol{e}_{d}^{n}\right\|_{1}^{2}+\left\|\boldsymbol{e}_{c}^{n+1}\right\|_{1}^{2}+\left\|\boldsymbol{e}_{c}^{n}\right\|_{1}\right)\right) I_{0}\left(\boldsymbol{u}^{n+1}, \boldsymbol{v}_{h}\right) \\
\leqslant & C\left(h^{2} I_{0}\left(\nabla p^{n+1}, \boldsymbol{\eta}_{h}\right)^{2}+h^{2} I_{1}\left(p^{n+1}, q_{h}\right)^{2}+\frac{1}{h^{2}} I_{0}\left(\boldsymbol{u}^{n+1}, \boldsymbol{v}_{h}\right)^{2}\right) \\
& +\frac{1}{4 \delta t}\left\|\boldsymbol{e}_{d}^{n+1}-\boldsymbol{e}_{d}^{n}\right\|_{0}^{2}+\frac{C}{\delta t} I_{0}\left(\boldsymbol{u}^{n+1}, \boldsymbol{v}_{h}\right)^{2}+\frac{\alpha-h^{2}}{3} G_{n+1}^{2} \\
& +\frac{v}{10}\left\|\boldsymbol{e}_{d}^{n+1}\right\|_{1}^{2}+C \frac{v^{1 / 2}}{h}\left\|\boldsymbol{e}_{d}^{n}\right\|_{1} I_{0}\left(\boldsymbol{u}^{n+1}, \boldsymbol{v}_{h}\right) \\
& +v\left(\left\|\boldsymbol{e}_{d}^{n+1}\right\|_{1}^{2}+\left\|\boldsymbol{e}_{d}^{n}\right\|_{1}^{2}+\left\|\boldsymbol{e}_{c}^{n+1}\right\|_{1}^{2}+\left\|\boldsymbol{e}_{c}^{n}\right\|_{1}^{2}\right) \frac{C}{h} I_{0}\left(\boldsymbol{u}^{n+1}, \boldsymbol{v}_{h}\right) .
\end{aligned}
$$

We split the convective terms the following way:

$$
\begin{aligned}
& c\left(\boldsymbol{u}_{h}^{n+\varepsilon}, \boldsymbol{u}_{h}^{n+1}, \boldsymbol{u}_{h}^{n+1}-\boldsymbol{v}_{h}\right)-c\left(\boldsymbol{u}^{n+\varepsilon}, \boldsymbol{u}^{n+1}, \boldsymbol{u}_{h}^{n+1}-\boldsymbol{v}_{h}\right) \\
& \quad=c\left(\boldsymbol{e}_{d}^{n+\varepsilon}, \boldsymbol{u}^{n+1}, \boldsymbol{u}_{h}^{n+1}-\boldsymbol{v}_{h}\right)+c\left(\boldsymbol{u}_{h}^{n+\varepsilon}, \boldsymbol{e}_{d}^{n+1}, \boldsymbol{u}_{h}^{n+1}-\boldsymbol{v}_{h}\right) \\
& \quad=-c\left(\boldsymbol{e}_{d}^{n+\varepsilon}, \boldsymbol{e}_{c}^{n+1}, \boldsymbol{u}_{h}^{n+1}-\boldsymbol{v}_{h}\right)+c\left(\boldsymbol{e}_{d}^{n+\varepsilon}, \boldsymbol{u}\left(t_{n+1}\right), \boldsymbol{u}_{h}^{n+1}-\boldsymbol{v}_{h}\right)
\end{aligned}
$$




$$
\begin{aligned}
& -c\left(\boldsymbol{e}_{d}^{n+\varepsilon}, \boldsymbol{e}_{d}^{n+1}, \boldsymbol{u}_{h}^{n+1}-\boldsymbol{v}_{h}\right)-c\left(\boldsymbol{e}_{c}^{n+\varepsilon}, \boldsymbol{e}_{d}^{n+1}, \boldsymbol{u}_{h}^{n+1}-\boldsymbol{v}_{h}\right) \\
& +c\left(\boldsymbol{u}\left(t_{n+\varepsilon}\right), \boldsymbol{e}_{d}^{n+1}, \boldsymbol{u}_{h}^{n+1}-\boldsymbol{v}_{h}\right) \\
= & c\left(\boldsymbol{e}_{d}^{n+\varepsilon}, \boldsymbol{e}_{c}^{n+1}, \boldsymbol{e}_{d}^{n+1}\right)-c\left(\boldsymbol{e}_{d}^{n+\varepsilon}, \boldsymbol{e}_{c}^{n+1}, \boldsymbol{u}^{n+1}-\boldsymbol{v}_{h}\right) \\
& -c\left(\boldsymbol{e}_{d}^{n+\varepsilon}, \boldsymbol{u}\left(t_{n+1}\right), \boldsymbol{e}_{d}^{n+1}\right)+c\left(\boldsymbol{e}_{d}^{n+\varepsilon}, \boldsymbol{u}\left(t_{n+1}\right), \boldsymbol{u}^{n+1}-\boldsymbol{v}_{h}\right) \\
& +c\left(\boldsymbol{e}_{d}^{n+\varepsilon}, \boldsymbol{e}_{d}^{n+1}, \boldsymbol{e}_{d}^{n+1}\right)-c\left(\boldsymbol{e}_{d}^{n+\varepsilon}, \boldsymbol{e}_{d}^{n+1}, \boldsymbol{u}^{n+1}-\boldsymbol{v}_{h}\right) \\
& +c\left(\boldsymbol{e}_{c}^{n+\varepsilon}, \boldsymbol{e}_{d}^{n+1}, \boldsymbol{e}_{d}^{n+1}\right)-c\left(\boldsymbol{e}_{c}^{n+\varepsilon}, \boldsymbol{e}_{d}^{n+1}, \boldsymbol{u}^{n+1}-\boldsymbol{v}_{h}\right) \\
& -c\left(\boldsymbol{u}\left(t_{n+\varepsilon}\right), \boldsymbol{e}_{d}^{n+1}, \boldsymbol{e}_{d}^{n+1}\right)+c\left(\boldsymbol{u}\left(t_{n+\varepsilon}\right), \boldsymbol{e}_{d}^{n+1}, \boldsymbol{u}^{n+1}-\boldsymbol{v}_{h}\right) .
\end{aligned}
$$

Due to the continuity properties of the trilinear form $c$, its skew symmetry in its last two arguments, the results of Theorem 1, the regularity assumed for the continuous solution $\boldsymbol{u}$ and Young's inequality, we have

$$
\begin{aligned}
& c\left(\boldsymbol{e}_{d}^{n+\varepsilon}, \boldsymbol{e}_{c}^{n+1}, \boldsymbol{e}_{d}^{n+1}\right) \leqslant C\left\|\boldsymbol{e}_{d}^{n+\varepsilon}\right\|_{1}\left\|\boldsymbol{e}_{c}^{n+1}\right\|_{1}\left\|\boldsymbol{e}_{d}^{n+1}\right\|_{1} \\
& \leqslant C \delta t^{1 / 2}\left\|\boldsymbol{e}_{d}^{n+\varepsilon}\right\|_{1}\left\|\boldsymbol{e}_{d}^{n+1}\right\|_{1} \\
& \leqslant \frac{v}{10}\left\|\boldsymbol{e}_{d}^{n+1}\right\|_{1}^{2}+C \delta t v\left\|\boldsymbol{e}_{d}^{n+\varepsilon}\right\|_{1}^{2} \\
& -c\left(\boldsymbol{e}_{d}^{n+\varepsilon}, \boldsymbol{e}_{c}^{n+1}, \boldsymbol{u}^{n+1}-\boldsymbol{v}_{h}\right) \leqslant C\left\|\boldsymbol{e}_{d}^{n+\varepsilon}\right\|_{1}\left\|\boldsymbol{e}_{c}^{n+1}\right\|_{1} I_{1}\left(\boldsymbol{u}^{n+1}, \boldsymbol{v}_{h}\right) \\
& \leqslant C \delta t^{1 / 2}\left\|\boldsymbol{e}_{d}^{n+\varepsilon}\right\|_{1} I_{1}\left(\boldsymbol{u}^{n+1}, \boldsymbol{v}_{h}\right) \\
& \leqslant \delta t v\left\|\boldsymbol{e}_{d}^{n+\varepsilon}\right\|_{1}^{2}+C I_{1}\left(\boldsymbol{u}^{n+1}, \boldsymbol{v}_{h}\right)^{2}, \\
& -c\left(\boldsymbol{e}_{d}^{n+\varepsilon}, \boldsymbol{u}\left(t_{n+1}\right), \boldsymbol{e}_{d}^{n+1}\right) \leqslant C\left\|\boldsymbol{e}_{d}^{n+\varepsilon}\right\|_{0}\left\|\boldsymbol{u}\left(t_{n+1}\right)\right\|_{2}\left\|\boldsymbol{e}_{d}^{n+1}\right\|_{1} \\
& \leqslant C\left\|\boldsymbol{e}_{d}^{n+\varepsilon}\right\|_{0}^{2}+\frac{v}{10}\left\|\boldsymbol{e}_{d}^{n+1}\right\|_{1}^{2}, \\
& c\left(\boldsymbol{e}_{d}^{n+\varepsilon}, \boldsymbol{u}\left(t_{n+1}\right), \boldsymbol{u}^{n+1}-\boldsymbol{v}_{h}\right) \leqslant C\left\|\boldsymbol{e}_{d}^{n+\varepsilon}\right\|_{0}\left\|\boldsymbol{u}\left(t_{n+1}\right)\right\|_{2} I_{1}\left(\boldsymbol{u}^{n+1}, \boldsymbol{v}_{h}\right) \\
& \leqslant C\left\|\boldsymbol{e}_{d}^{n+\varepsilon}\right\|_{0}^{2}+I_{1}\left(\boldsymbol{u}^{n+1}, \boldsymbol{v}_{h}\right)^{2} \\
& c\left(\boldsymbol{e}_{d}^{n+\varepsilon}, \boldsymbol{e}_{d}^{n+1}, \boldsymbol{e}_{d}^{n+1}\right)=0, \\
& -c\left(\boldsymbol{e}_{d}^{n+\varepsilon}, \boldsymbol{e}_{d}^{n+1}, \boldsymbol{u}^{n+1}-\boldsymbol{v}_{h}\right) \leqslant C\left\|\boldsymbol{e}_{d}^{n+\varepsilon}\right\|_{1}\left\|\boldsymbol{e}_{d}^{n+1}\right\|_{1} I_{1}\left(\boldsymbol{u}^{n+1}, \boldsymbol{v}_{h}\right) \\
& \leqslant \frac{v}{10}\left\|\boldsymbol{e}_{d}^{n+1}\right\|_{1}^{2}+C v\left\|\boldsymbol{e}_{d}^{n+\varepsilon}\right\|_{1}^{2} I_{1}\left(\boldsymbol{u}^{n+1}, \boldsymbol{v}_{h}\right)^{2}, \\
& c\left(\boldsymbol{e}_{c}^{n+\varepsilon}, \boldsymbol{e}_{d}^{n+1}, \boldsymbol{e}_{d}^{n+1}\right)=0, \\
& -c\left(\boldsymbol{e}_{c}^{n+\varepsilon}, \boldsymbol{e}_{d}^{n+1}, \boldsymbol{u}^{n+1}-\boldsymbol{v}_{h}\right) \leqslant C\left\|\boldsymbol{e}_{c}^{n+\varepsilon}\right\|_{1}\left\|\boldsymbol{e}_{d}^{n+1}\right\|_{1} I_{1}\left(\boldsymbol{u}^{n+1}, \boldsymbol{v}_{h}\right) \\
& \leqslant \frac{v}{10}\left\|\boldsymbol{e}_{d}^{n+1}\right\|_{1}^{2}+C v\left\|\boldsymbol{e}_{c}^{n+\varepsilon}\right\|_{1}^{2} I_{1}\left(\boldsymbol{u}^{n+1}, \boldsymbol{v}_{h}\right)^{2}, \\
& -c\left(\boldsymbol{u}\left(t_{n+\varepsilon}\right), \boldsymbol{e}_{d}^{n+1}, \boldsymbol{e}_{d}^{n+1}\right)=0, \\
& c\left(\boldsymbol{u}\left(t_{n+\varepsilon}\right), \boldsymbol{e}_{d}^{n+1}, \boldsymbol{u}^{n+1}-\boldsymbol{v}_{h}\right) \leqslant C\left\|\boldsymbol{u}\left(t_{n+\varepsilon}\right)\right\|_{2}\left\|\boldsymbol{e}_{d}^{n+1}\right\|_{1} I_{0}\left(\boldsymbol{u}^{n+1}, \boldsymbol{v}_{h}\right) \\
& \leqslant \frac{v}{10}\left\|\boldsymbol{e}_{d}^{n+1}\right\|_{1}^{2}+C I_{0}\left(\boldsymbol{u}^{n+1}, \boldsymbol{v}_{h}\right)^{2} .
\end{aligned}
$$


Taking all the previous inequalities into account, and using (20), we find

$$
\begin{aligned}
\left\|\boldsymbol{e}_{d}^{n+1}\right\|_{0}^{2}- & \left\|\boldsymbol{e}_{d}^{n}\right\|_{0}^{2}+\left\|\boldsymbol{e}_{d}^{n+1}-\boldsymbol{e}_{d}^{n}\right\|_{0}^{2}+v \delta t\left\|\boldsymbol{e}_{d}^{n+1}\right\|_{1}^{2}+\delta t h^{2} G_{n+1}^{2} \\
\leqslant & C \delta t\left(I_{1}\left(\boldsymbol{u}^{n+1}, \boldsymbol{v}_{h}\right)^{2}+h^{2} I_{1}\left(p^{n+1}, q_{h}\right)^{2}+h^{2} I_{0}\left(\nabla p^{n+1}, \boldsymbol{\eta}_{h}\right)^{2}\right. \\
& \left.+\frac{1}{h^{2}} I_{0}\left(\boldsymbol{u}^{n+1}, \boldsymbol{v}_{h}\right)^{2}+I_{0}\left(p^{n+1}, q_{h}\right)^{2}\right)+C I_{0}\left(\boldsymbol{u}^{n+1}, \boldsymbol{v}_{h}\right)^{2} \\
& +\delta t v\left(\left\|\boldsymbol{e}_{d}^{n+1}\right\|_{1}^{2}+\left\|\boldsymbol{e}_{d}^{n}\right\|_{1}^{2}\right) \frac{C}{h} I_{0}\left(\boldsymbol{u}^{n+1}, \boldsymbol{v}_{h}\right)+\delta t v\left(\left\|\boldsymbol{e}_{c}^{n+1}\right\|_{1}^{2}+\left\|\boldsymbol{e}_{c}^{n}\right\|_{1}^{2}\right) \frac{C}{h} I_{0}\left(\boldsymbol{u}^{n+1}, \boldsymbol{v}_{h}\right) \\
& +C \delta t^{2} v\left\|\boldsymbol{e}_{d}^{n+\varepsilon}\right\|_{1}^{2}+C \delta t\left\|\boldsymbol{e}_{d}^{n+\varepsilon}\right\|_{0}^{2}+C \frac{\delta t v^{1 / 2}}{h}\left\|\boldsymbol{e}_{d}^{n}\right\|_{1} I_{0}\left(\boldsymbol{u}^{n+1}, \boldsymbol{v}_{h}\right) \\
& +C \delta t v\left\|\boldsymbol{e}_{d}^{n+\varepsilon}\right\|_{1}^{2} I_{1}\left(\boldsymbol{u}^{n+1}, \boldsymbol{v}_{h}\right)^{2}+C \delta t v\left\|\boldsymbol{e}_{c}^{n+\varepsilon}\right\|_{1}^{2} I_{1}\left(\boldsymbol{u}^{n+1}, \boldsymbol{v}_{h}\right)^{2} .
\end{aligned}
$$

Taking the infimum with respect to $\left(\boldsymbol{v}_{h}, q_{h}, \boldsymbol{\eta}_{h}\right) \in V_{h, 0} \times Q_{h} \times V_{h}$, we get

$$
\begin{aligned}
\| \boldsymbol{e}_{d}^{n+1} & \left\|_{0}^{2}-\right\| \boldsymbol{e}_{d}^{n}\left\|_{0}^{2}+\right\| \boldsymbol{e}_{d}^{n+1}-\boldsymbol{e}_{d}^{n}\left\|_{0}^{2}+v \delta t\right\| \boldsymbol{e}_{d}^{n+1} \|_{1}^{2}+\delta t h^{2} G_{n+1}^{2} \\
\leqslant & C \delta t\left(E_{n}(h)\right)^{2}+C h^{2}\left(E_{n}(h)\right)^{2} \\
& +C \delta t v\left(\left\|\boldsymbol{e}_{d}^{n+1}\right\|_{1}^{2}+\left\|\boldsymbol{e}_{d}^{n}\right\|_{1}^{2}+\left\|\boldsymbol{e}_{c}^{n+1}\right\|_{1}^{2}+\left\|\boldsymbol{e}_{c}^{n}\right\|_{1}^{2}\right) E_{n}(h) \\
& +C \delta t^{2} v\left\|\boldsymbol{e}_{d}^{n+\varepsilon}\right\|_{1}^{2}+C \delta t\left\|\boldsymbol{e}_{d}^{n+\varepsilon}\right\|_{0}^{2}+C \delta t v^{1 / 2}\left\|\boldsymbol{e}_{d}^{n}\right\|_{1} E_{n}(h) \\
& +C \delta t v\left(\left\|\boldsymbol{e}_{d}^{n+\varepsilon}\right\|_{1}^{2}+\left\|\boldsymbol{e}_{c}^{n+\varepsilon}\right\|_{1}^{2}\right)\left(E_{n}(h)\right)^{2} .
\end{aligned}
$$

Adding up (34) from $n=0$ to $N$, using assumption $\mathrm{H} 4$, the definition of $E(h)$ and the estimates of Theorem 1, we get

$$
\begin{aligned}
&\left\|\boldsymbol{e}_{d}^{N+1}\right\|_{0}^{2}+\sum_{n=0}^{N}\left\|\boldsymbol{e}_{d}^{n+1}-\boldsymbol{e}_{d}^{n}\right\|_{0}^{2}+v \delta t \sum_{n=0}^{N}\left\|\boldsymbol{e}_{d}^{n+1}\right\|_{1}^{2}+\delta t h^{2} \sum_{n=0}^{N} G_{n+1}^{2} \\
& \leqslant C(E(h))^{2}+C\left(v \delta t \sum_{n=0}^{N}\left\|\boldsymbol{e}_{d}^{n+1}\right\|_{1}^{2}\right) E(h)+C\left(v \delta t \sum_{n=0}^{N}\left\|\boldsymbol{e}_{c}^{n+1}\right\|_{1}^{2}\right) E(h) \\
&+C v \delta t^{2} \sum_{n=0}^{N}\left\|\boldsymbol{e}_{d}^{n+\varepsilon}\right\|_{1}^{2}+C \delta t \sum_{n=0}^{N}\left\|\boldsymbol{e}_{d}^{n+\varepsilon}\right\|_{0}^{2}+C\left(\delta t v^{1 / 2} \sum_{n=0}^{N}\left\|\boldsymbol{e}_{d}^{n}\right\|_{1}\right) E(h) \\
&+C\left(v \delta t \sum_{n=0}^{N}\left\|\boldsymbol{e}_{d}^{n+\varepsilon}\right\|_{1}^{2}+v \delta t \sum_{n=0}^{N}\left\|\boldsymbol{e}_{c}^{n+\varepsilon}\right\|_{1}^{2}\right)(E(h))^{2} \\
& \leqslant C(E(h))^{2}+C\left(v \delta t \sum_{n=0}^{N}\left\|\boldsymbol{e}_{d}^{n+1}\right\|_{1}^{2}\right) E(h)+C \delta t^{2} E(h) \\
&+C \nu \delta t^{2} \sum_{n=0}^{N}\left\|\boldsymbol{e}_{d}^{n+\varepsilon}\right\|_{1}^{2}+C \delta t \sum_{n=0}^{N}\left\|\boldsymbol{e}_{d}^{n+\varepsilon}\right\|_{0}^{2}+C\left(\delta t v \sum_{n=0}^{N}\left\|\boldsymbol{e}_{d}^{n}\right\|_{1}^{2}\right)^{1 / 2} E(h)
\end{aligned}
$$




$$
\begin{aligned}
\leqslant & C(E(h))^{2}+C\left(v \delta t \sum_{n=0}^{N}\left\|\boldsymbol{e}_{d}^{n+1}\right\|_{1}^{2}\right) E(h)+C \delta t^{2} E(h) \\
& +C v \delta t^{2} \sum_{n=0}^{N}\left\|\boldsymbol{e}_{d}^{n+\varepsilon}\right\|_{1}^{2}+C \delta t \sum_{n=0}^{N}\left\|\boldsymbol{e}_{d}^{n+\varepsilon}\right\|_{0}^{2}+\frac{1}{2}\left(\delta t v \sum_{n=0}^{N}\left\|\boldsymbol{e}_{d}^{n+1}\right\|_{1}^{2}\right)
\end{aligned}
$$

since $E(h) \leqslant C h,(E(h))^{2} \leqslant E(h)$ for $h$ small enough and $|Z|_{l^{1}(X)} \leqslant C|Z|_{l^{2}(X)}$ for any $Z$ and $X$ (see Remark 1). For sufficiently small $h$, the second term in the right hand side can be passed over to the left hand side, since $E(h)$ tends to 0 as $h$ tends to 0 . By the discrete Gronwall inequality, this implies, for sufficiently small $\delta t$ in the case $\varepsilon=1$,

$$
\left\|\boldsymbol{e}_{d}^{N+1}\right\|_{0}^{2}+\sum_{n=0}^{N}\left\|\boldsymbol{e}_{d}^{n+1}-\boldsymbol{e}_{d}^{n}\right\|_{0}^{2}+v \delta t \sum_{n=0}^{N}\left\|\boldsymbol{e}_{d}^{n+1}\right\|_{1}^{2}+\delta t h^{2} \sum_{n=0}^{N} G_{n+1}^{2} \leqslant C(E(h))^{2}+C \delta t^{2} E(h)
$$

and (33) follows.

Remark 3. For equal order interpolations of degree $k$, the spatial error function $E(h)$ behaves like $h^{k}$, the worst case being that of linear $\left(P_{1}\right)$ and multilinear $\left(Q_{1}\right)$ elements. In general, one always has $E(h) \leqslant C h$; due to assumption (32), this result proves in particular that the discrete velocities are bounded in $l^{\infty}\left(\boldsymbol{H}_{0}^{1}(\Omega)\right)$ by a constant independent of $\delta t$ and $h$, since

$$
\begin{aligned}
\left\|\boldsymbol{u}_{h}^{n+1}\right\|_{1} & \leqslant\left\|\boldsymbol{u}\left(t_{n+1}\right)\right\|_{1}+\left\|\boldsymbol{e}_{c}^{n+1}\right\|_{1}+\left\|\boldsymbol{e}_{d}^{n+1}\right\|_{1} \\
& \leqslant C\left(1+\delta t^{1 / 2}+\left(\frac{(E(h))^{2}}{\delta t}\right)^{1 / 2}\right) \leqslant C\left(1+\left(\frac{h^{2}}{\delta t}\right)^{1 / 2}\right) \leqslant C .
\end{aligned}
$$

This is the key point to obtain improved stability estimates in the next section.

Remark 4. The last term in the estimate (33) for the discrete velocity is due to the presence of the convective term in the equations (it is not present in an analysis of the linear Stokes case) and arises from the estimates of the semidiscrete problem. Again, since $E(h) \leqslant C h$, this extra term is always smaller than $\delta t^{2}$, and the method remains first order accurate in time for the velocity.

\subsection{Improved stability estimate}

As a consequence of the convergence analysis of the previous section, the stability results of Section 3.2 can be improved as follows:

Proposition 2. Assume (R1), (R2), (R4) and H1-H4 hold; then, there exists a constant $C>0$ independent of $\delta t$ and $h$ such that, for small enough $h$ and, if $\varepsilon=1$, small enough $\delta t$,

$$
\delta t h^{2} \sum_{n=0}^{N}\left\|\nabla p_{h}^{n+1}\right\|_{0}^{2} \leqslant C
$$

Proof. In a similar way to [7], taking $\boldsymbol{v}=\boldsymbol{u}_{h}^{n+1}$ in (8) (with $\theta=1$ ), $q_{h}=p_{h}^{n+1}$ in (9) and $\boldsymbol{\eta}_{h}=\alpha \boldsymbol{\xi}_{h}^{n+1}$ in (10), and adding them up, we get

$$
\left(\frac{\boldsymbol{u}_{h}^{n+1}-\boldsymbol{u}_{h}^{n}}{\delta t}, \boldsymbol{u}_{h}^{n+1}\right)+v\left\|\nabla \boldsymbol{u}_{h}^{n+1}\right\|_{0}^{2}+\alpha\left\|\nabla p_{h}^{n+1}-\boldsymbol{\xi}_{h}^{n+1}\right\|_{0}^{2}=\left(\boldsymbol{f}^{n+1}, \boldsymbol{u}_{h}^{n+1}\right) .
$$


From (36), it is found that

$$
\begin{aligned}
& \left\|\boldsymbol{u}_{h}^{N+1}\right\|_{0}^{2}+\sum_{n=0}^{N}\left\|\boldsymbol{u}_{h}^{n+1}-\boldsymbol{u}_{h}^{n}\right\|_{0}^{2}+v \delta t \sum_{n=0}^{N}\left\|\boldsymbol{u}_{h}^{n+1}\right\|_{1}^{2}+\alpha \delta t \sum_{n=0}^{N}\left\|\nabla p_{h}^{n+1}-\boldsymbol{\xi}_{h}^{n+1}\right\|_{0}^{2} \\
& \leqslant C\left(\delta t \sum_{n=0}^{N}\left\|\boldsymbol{f}^{n+1}\right\|_{0}^{2}+1\right) \leqslant C\left(\int_{0}^{T}\|\boldsymbol{f}(t)\|_{0}^{2} \mathrm{~d} t+1\right) .
\end{aligned}
$$

Thus, the third component $P_{h, 3}\left(\nabla p_{h}^{n+1}\right)=\nabla p_{h}^{n+1}-\xi_{h}^{n+1}$ in the decomposition of $\nabla p_{h}^{n+1}$ in $E_{h}$ is bounded; due to assumption (23), it only remains to bound $P_{h, 1}\left(\nabla p_{h}^{n+1}\right)$, which belongs to $V_{h, 0}$. Using the continuity of the forms $a$ and $c$, the inverse estimate (22) and the result of Remark 2, we have

$$
\begin{aligned}
\left\|P_{h, 1}\left(\nabla p_{h}^{n+1}\right)\right\|_{0}^{2}= & \left(\nabla p_{h}^{n+1}, P_{h, 1}\left(\nabla p_{h}^{n+1}\right)\right) \\
= & -\left(\frac{\boldsymbol{u}_{h}^{n+1}-\boldsymbol{u}_{h}^{n}}{\delta t}, P_{h, 1}\left(\nabla p_{h}^{n+1}\right)\right)-a\left(\boldsymbol{u}_{h}^{n+1}, P_{h, 1}\left(\nabla p_{h}^{n+1}\right)\right) \\
& \quad-c\left(\boldsymbol{u}_{h}^{n+\varepsilon}, \boldsymbol{u}_{h}^{n+1}, P_{h, 1}\left(\nabla p_{h}^{n+1}\right)\right)+\left(\boldsymbol{f}^{n+1}, P_{h, 1}\left(\nabla p_{h}^{n+1}\right)\right) \\
\leqslant & \left\|P_{h, 1}\left(\nabla p_{h}^{n+1}\right)\right\|_{0}\left(\frac{1}{\delta t}\left\|\boldsymbol{u}_{h}^{n+1}-\boldsymbol{u}_{h}^{n}\right\|_{0}+\left\|\boldsymbol{f}^{n+1}\right\|_{0}\right. \\
& \left.\quad+\frac{C v}{h}\left\|\boldsymbol{u}_{h}^{n+1}\right\|_{1}+\frac{C}{h}\left\|\boldsymbol{u}_{h}^{n+\varepsilon}\right\|_{1}\left\|\boldsymbol{u}_{h}^{n+1}\right\|_{1}\right) \\
\leqslant & \left\|P_{h, 1}\left(\nabla p_{h}^{n+1}\right)\right\|_{0}\left(\frac{1}{\delta t}\left\|\boldsymbol{u}_{h}^{n+1}-\boldsymbol{u}_{h}^{n}\right\|_{0}+\left\|\boldsymbol{f}^{n+1}\right\|_{0}+\frac{C}{h}\right) .
\end{aligned}
$$

Dividing this estimate by $\left\|P_{h, 1}\left(\nabla p_{h}^{n+1}\right)\right\|_{0}$, squaring the result, multiplying by $\delta t h^{2}$ and adding up for $n=0, \ldots, N$, we find

$$
\delta t h^{2} \sum_{n=0}^{N}\left\|\nabla p_{h}^{n+1}\right\|_{0}^{2} \leqslant C\left(\frac{h^{2}}{\delta t} \sum_{n=0}^{N}\left\|\boldsymbol{u}_{h}^{n+1}-\boldsymbol{u}_{h}^{n}\right\|_{0}+1\right) \leqslant C
$$

due to the assumed behaviour (32) on the time step size.

\subsection{Error estimates for the pressure}

We begin this section with an estimate for the discrete pressure gradient:

Proposition 3. Assume (R1), (R2), (R3), (R4) and $\mathrm{H} 1-\mathrm{H} 4$ hold; then, there exists a constant $C>0$ independent of $\delta t$ and $h$ such that, for small enough $h$ and, if $\varepsilon=1$, small enough $\delta t$,

$$
\delta t h^{2} \sum_{n=0}^{N}\left\|\nabla r_{d}^{n+1}\right\|_{0}^{2} \leqslant C\left((E(h))^{2}+E(h) \delta t^{2}\right) .
$$

Proof. From Lemma 1, we have

$$
\begin{aligned}
& \left\|\nabla r_{d}^{n+1}\right\|_{0}^{2} \\
& \quad \leqslant C\left\{\left(I_{0}\left(\nabla p^{n+1}, \boldsymbol{\eta}_{h}\right)\right)^{2}+\left(I_{1}\left(p^{n+1}, q_{h}\right)\right)^{2}+\frac{1}{\delta t^{2}}\left\|\boldsymbol{e}_{d}^{n+1}-\boldsymbol{e}_{d}^{n}\right\|_{0}^{2}+\frac{v}{h^{2}}\left\|\boldsymbol{e}_{d}^{n+1}\right\|_{1}^{2}+\left(G_{n+1}\right)^{2}\right\} .
\end{aligned}
$$


Thus,

$$
\begin{aligned}
\delta t h^{2} \sum_{n=0}^{N}\left\|\nabla r_{d}^{n+1}\right\|_{0}^{2} \leqslant C\{ & \delta t h^{2} \sum_{n=0}^{N}\left(I_{0}\left(\nabla p^{n+1}, \boldsymbol{\eta}_{h}\right)\right)^{2}+\delta t h^{2} \sum_{n=0}^{N}\left(I_{1}\left(p^{n+1}, q_{h}\right)\right)^{2} \\
& \left.+\frac{h^{2}}{\delta t}\left\|\boldsymbol{e}_{d}^{n+1}-\boldsymbol{e}_{d}^{n}\right\|_{0}^{2}+v \delta t \sum_{n=0}^{N}\left\|\boldsymbol{e}_{d}^{n+1}\right\|_{1}^{2}+\delta t h^{2} \sum_{n=0}^{N}\left(G_{n+1}\right)^{2}\right\} .
\end{aligned}
$$

Taking the infimum with respect to $\eta_{h}$ and $q_{h}$ and using (32), this implies

$$
\delta t h^{2} \sum_{n=0}^{N}\left\|\nabla r_{d}^{n+1}\right\|_{0}^{2} \leqslant C\left\{\delta t \sum_{n=0}^{N}\left(E_{n}(h)\right)^{2}+\sum_{n=0}^{N}\left\|\boldsymbol{e}_{d}^{n+1}-\boldsymbol{e}_{d}^{n}\right\|_{0}^{2}+v \delta t \sum_{n=0}^{N}\left\|\boldsymbol{e}_{d}^{n+1}\right\|_{1}^{2}+\delta t h^{2} \sum_{n=0}^{N}\left(G_{n+1}\right)^{2}\right\},
$$

and (38) follows from (35) and the definition of $E(h),(29)$.

Since we have obtained error estimates for the fully discrete pressure gradient and the semidiscrete pressure itself, we now present some estimates for the fully discrete pressure solution, which are based on a classical duality argument:

Proposition 4. Assume (R1)-(R4) and $\mathrm{H} 1-\mathrm{H} 4$ hold; then, there exists a constant $C>0$ independent of $\delta t$ and $h$ such that, for small enough $h$ and, if $\varepsilon=1$, small enough $\delta t$,

$$
\delta t^{2} \sum_{n=0}^{N}\left\|r_{d}^{n+1}\right\|_{0}^{2} \leqslant C\left((E(h))^{2}+\delta t^{2}\right)
$$

Proof. Let $z \in \boldsymbol{H}_{0}^{1}(\Omega)$ and $\xi \in L_{0}^{2}(\Omega)$ be the solution of the following Stokes problem:

$$
\begin{array}{ll}
-\Delta z+\nabla \xi=0 & \text { in } \Omega, \\
\nabla \cdot z=r_{d}^{n+1} & \text { in } \Omega, \\
z=0 & \text { on } \Gamma .
\end{array}
$$

Standard results for this problem yield

$$
\|z\|_{1} \leqslant C\left\|r_{d}^{n+1}\right\|_{0}, \quad\|\xi\|_{0} \leqslant C\left\|r_{d}^{n+1}\right\|_{0} .
$$

If $\boldsymbol{z}_{h} \in V_{h, 0}$ now satisfies

$$
\left\|z-z_{h}\right\|_{m} \leqslant C h^{1-m}\|z\|_{1}
$$

for $m=0,1$, we have

$$
\begin{aligned}
\left\|r_{d}^{n+1}\right\|_{0}^{2}= & \left(r_{d}^{n+1}, r_{d}^{n+1}\right)=\left(\nabla \cdot \boldsymbol{z}, r_{d}^{n+1}\right)=-\left(\boldsymbol{z}, \nabla r_{d}^{n+1}\right) \\
= & -\left(\boldsymbol{z}-\boldsymbol{z}_{h}, \nabla r_{d}^{n+1}\right)-\left(\boldsymbol{z}_{h}, \nabla r_{d}^{n+1}\right) \\
= & -\left(\boldsymbol{z}-\boldsymbol{z}_{h}, \nabla r_{d}^{n+1}\right)+\left(\frac{\boldsymbol{e}_{d}^{n+1}-\boldsymbol{e}_{d}^{n}}{\delta t}, \boldsymbol{z}_{h}\right)+v\left(\nabla \boldsymbol{e}_{d}^{n+1}, \nabla \boldsymbol{z}_{h}\right) \\
& -c\left(\boldsymbol{u}_{h}^{n+\varepsilon}, \boldsymbol{u}_{h}^{n+1}, \boldsymbol{z}_{h}\right)+c\left(\boldsymbol{u}^{n+\varepsilon}, \boldsymbol{u}^{n+1}, \boldsymbol{z}_{h}\right) .
\end{aligned}
$$


Furthermore,

$$
\begin{aligned}
&-\left(\boldsymbol{z}-\boldsymbol{z}_{h}, \nabla r_{d}^{n+1}\right) \leqslant\left\|\boldsymbol{z}-\boldsymbol{z}_{h}\right\|_{0}\left\|\nabla r_{d}^{n+1}\right\|_{0} \leqslant C h\left\|\nabla r_{d}^{n+1}\right\|_{0}\|\boldsymbol{z}\|_{1} \leqslant C h\left\|\nabla r_{d}^{n+1}\right\|_{0}\left\|r_{d}^{n+1}\right\|_{0}, \\
&\left(\frac{\boldsymbol{e}_{d}^{n+1}-\boldsymbol{e}_{d}^{n}}{\delta t}, z_{h}\right) \leqslant \frac{1}{\delta t}\left\|\boldsymbol{e}_{d}^{n+1}-\boldsymbol{e}_{d}^{n}\right\|_{0}\left\|\boldsymbol{z}_{h}\right\|_{0} \leqslant \frac{1}{\delta t}\left\|\boldsymbol{e}_{d}^{n+1}-\boldsymbol{e}_{d}^{n}\right\|_{0}\left(\left\|z-\boldsymbol{z}_{h}\right\|_{0}+\|\boldsymbol{z}\|_{0}\right) \\
& \leqslant \frac{1}{\delta t}\left\|\boldsymbol{e}_{d}^{n+1}-\boldsymbol{e}_{d}^{n}\right\|_{0}\left(C h\|\boldsymbol{z}\|_{1}+C\|\boldsymbol{z}\|_{1}\right) \leqslant \frac{1}{\delta t}\left\|\boldsymbol{e}_{d}^{n+1}-\boldsymbol{e}_{d}^{n}\right\|_{0}\left\|r_{d}^{n+1}\right\|_{0}, \\
& v\left(\nabla \boldsymbol{e}_{d}^{n+1}, \nabla \boldsymbol{z}_{h}\right) \leqslant C v^{1 / 2}\left\|\boldsymbol{e}_{d}^{n+1}\right\|_{1}\left\|\boldsymbol{z}_{h}\right\|_{1} \leqslant C v^{1 / 2}\left\|\boldsymbol{e}_{d}^{n+1}\right\|_{1}\left\|r_{d}^{n+1}\right\|_{0}, \\
&-c\left(\boldsymbol{u}_{h}^{n+\varepsilon}, \boldsymbol{u}_{h}^{n+1}, \boldsymbol{z}_{h}\right)+c\left(\boldsymbol{u}^{n+\varepsilon}, \boldsymbol{u}^{n+1}, \boldsymbol{z}_{h}\right)=c\left(\boldsymbol{u}^{n+\varepsilon}, \boldsymbol{e}_{d}^{n+1}, \boldsymbol{z}_{h}\right)+c\left(\boldsymbol{e}_{d}^{n+\varepsilon}, \boldsymbol{u}_{h}^{n+1}, \boldsymbol{z}_{h}\right) \\
& \leqslant C\left(\left\|\boldsymbol{u}^{n+\varepsilon}\right\|_{1}\left\|\boldsymbol{e}_{d}^{n+1}\right\|_{1}+\left\|\boldsymbol{e}_{d}^{n+\varepsilon}\right\|_{1}\left\|\boldsymbol{u}_{h}^{n+1}\right\|_{1}\right)\left\|\boldsymbol{z}_{h}\right\|_{1} \\
& \leqslant C\left(\left\|\boldsymbol{e}_{d}^{n+1}\right\|_{1}+\left\|\boldsymbol{e}_{d}^{n+\varepsilon}\right\|_{1}\right)\left(\left\|\boldsymbol{z}-\boldsymbol{z}_{h}\right\|_{1}+\|\boldsymbol{z}\|_{1}\right) \\
& \leqslant C v^{1 / 2}\left(\left\|\boldsymbol{e}_{d}^{n+1}\right\|_{1}+\left\|\boldsymbol{e}_{d}^{n+\varepsilon}\right\|_{1}\right)\left\|r_{d}^{n+1}\right\|_{0} .
\end{aligned}
$$

Estimate (39) is obtained dividing by $\left\|r_{d}^{n+1}\right\|_{0}$ throughout, squaring the result, multiplying by $\delta t^{2}$ and adding up from $n=0$ to $N$, due to (35) and (38).

\subsection{Global error behaviour}

As a consequence of the previous results, we have:

Corolary 1. Assume (R1)-(R4) and $\mathrm{H} 1-\mathrm{H} 4$ hold; assume also that, for $n=0, \ldots, N, \boldsymbol{u}^{n+1} \in \boldsymbol{H}^{r}(\Omega)$, $r \geqslant 2$ and $p^{n+1} \in H^{s}(\Omega), s \geqslant 1$, and that they are uniformly bounded in these spaces. Then, there exists a constant $C>0$ independent of $\delta t$ and $h$ such that, for small enough $h$ and, if $\varepsilon=1$, small enough $\delta t$,

$$
\left\|\boldsymbol{e}^{N+1}\right\|_{0}^{2}+v \delta t \sum_{n=0}^{N}\left\|\boldsymbol{e}^{n+1}\right\|_{1}^{2}+\delta t^{2} \sum_{n=0}^{N}\left\|r^{n+1}\right\|_{0}^{2} \leqslant C\left(\delta t^{2}+h^{2 k}\right),
$$

where $k=\min \left(r-1, s, k_{v}, k_{q}+1\right)$.

Proof. This estimate follows from Theorems 1 and 3, Propositions 1 and 4, assumption (32), the regularity assumed of the semidiscrete solution $\left(\boldsymbol{u}^{n+1}, p^{n+1}\right)$ and the approximating properties of the finite element spaces considered.

Remark 5. The condition $\delta t \geqslant C h^{2}$ arises due to the proof technique employed, which deals with the temporal error first and then the spatial error. However, according to the results of Corollary 1, accuracy considerations indicate that, when equal order interpolation of degree $k$ is used, $\delta t$ should be of order $h^{k}$; for linear $\left(P_{1}\right)$ and bilinear $\left(Q_{1}\right)$ elements, one has $k=1$, so that assumption $\mathrm{H} 4$ is fulfilled. Even for quadratic $\left(P_{2}\right)$ and biquadratic $\left(Q_{2}\right)$ elements, one still has $k=2$, making $\mathrm{H} 4$ acceptable.

\section{References}

[1] K. Boukir, Y. Maday, B. Métivet, E. Razafindrakoto, A high-order characteristic/finite element method for the incompressible Navier-Stokes equations, Internat. J. Numer. Methods Fluids 25 (1997) 1421-1454. 
[2] S.C. Brenner, L.R. Scott, The Mathematical Theory of Finite Element Methods, Springer, Berlin, 1994.

[3] F. Brezzi, R.S. Falk, Stability of higher-order Hood-Taylor methods, SIAM J. Numer. Anal. 28 (1991) 581590.

[4] R. Codina, A stabilized finite element method for generalized stationary incompressible flows, Comput. Methods Appl. Mech. Eng. 190 (2001) 2681-2706.

[5] R. Codina, J. Blasco, A finite element formulation for the Stokes problem allowing equal velocity-pressure interpolation, Comput. Methods Appl. Mech. Eng. 143 (1997) 373-391.

[6] R. Codina, J. Blasco, Analysis of a pressure stabilized finite element approximation of the stationary NavierStokes equations, Numer. Math. 87 (2000) 59-81.

[7] R. Codina, J. Blasco, Stabilized finite element method for the transient Navier-Stokes equations based on a pressure gradient projection, Comput. Methods Appl. Mech. Eng. 182 (2000) 277-300.

[8] Constantin P. Foias, Navier-Stokes Equations, Chicago Lectures in Mathematics, The University of Chicago Press, Chicago and London, 1988.

[9] L.P. Franca, S.L. Frey, Stabilized finite element methods: II. The incompressible Navier-Stokes equations, Comput. Methods Appl. Mech. Eng. 99 (1992) 209-233.

[10] L.P. Franca, T.J.R. Hughes, Convergence analysis of Galerkin least-squares methods for symmetric advectivediffusive forms of the Stokes and incompressible Navier-Stokes equations, Comput. Methods Appl. Mech. Eng. 105 (1993) 285-298.

[11] V. Girault, P.A. Raviart, Finite Element Approximation of the Navier-Stokes Equation, Springer, New York, 1986.

[12] J.-L. Guermond, L. Quartapelle, On stability and convergence of projection methods based on pressure Poisson equation, Internat. J. Numer. Methods Fluids 26 (1998) 1039-1053.

[13] P. Hansbo, A. Szepessy, A velocity-pressure streamline diffusion finite element method for the incompressible Navier-Stokes equations, Comput. Methods Appl. Mech. Eng. 84 (1990) 175-192.

[14] J.G. Heywood, R. Rannacher, Finite element approximation of the nonstationary Navier-Stokes problem: Part 1: Regularity of solutions and second order error estimates for spatial discretization, SIAM J. Numer. Anal. 19 (1982) 275-311.

[15] J.G. Heywood, R. Rannacher, Finite element approximation of the nonstationary Navier-Stokes problem: Part 4: Error analysis for second-order time discretization, SIAM J. Numer. Anal. 27 (1990) 353-384.

[16] T.J.R. Hughes, Multiscale phenomena: Greens functions, subgrid scale models, bubbles and the origins of stabilized methods, Comput. Methods Appl. Mech. Eng. 127 (1995) 387-401.

[17] T.J.R. Hughes, L.P. Franca, M. Balestra, A new finite element formulation for computational fluid dynamics: V. Circumventing the Babuška-Brezzi condition: a stable Petrov-Galerkin formulation of the Stokes problem accommodating equal-order interpolations, Comput. Methods Appl. Mech. Eng. 59 (1986) 85-99.

[18] T.J.R. Hughes, L.P. Franca, G.M. Hulbert, A new finite element formulation for computational fluid dynamics: VIII. The Galerkin/least squares method for advective-diffusive equations, Comput. Methods Appl. Mech. Eng. 73 (1989) 173-189.

[19] G. Lube, D. Weiss, Stabilized finite element methods for singularly perturbed parabolic problems, Appl. Numer. Math. 17 (1995) 431-459.

[20] J. Shen, On error estimates of projection methods for Navier-Stokes equations: first-order schemes, SIAM J. Numer. Anal. 29 (1992) 57-77.

[21] J. Shen, Remarks on the pressure error estimates for the projection method, Numer. Math. 67 (1994) 513-520.

[22] R. Stenberg, A technique for analyzing finite element methods for viscous incompressible flow, Internat. J. Numer. Methods Fluids 11 (1990) 935-948.

[23] G. Strang, J. Fix, An Analysis of the Finite Element Method, Prentice-Hall, Englewood Cliffs, NJ, 1973.

[24] R. Temam, Navier-Stokes Equations, Theory and Numerical Analysis, 3rd Edition, North-Holland, Amsterdam, 1984. 